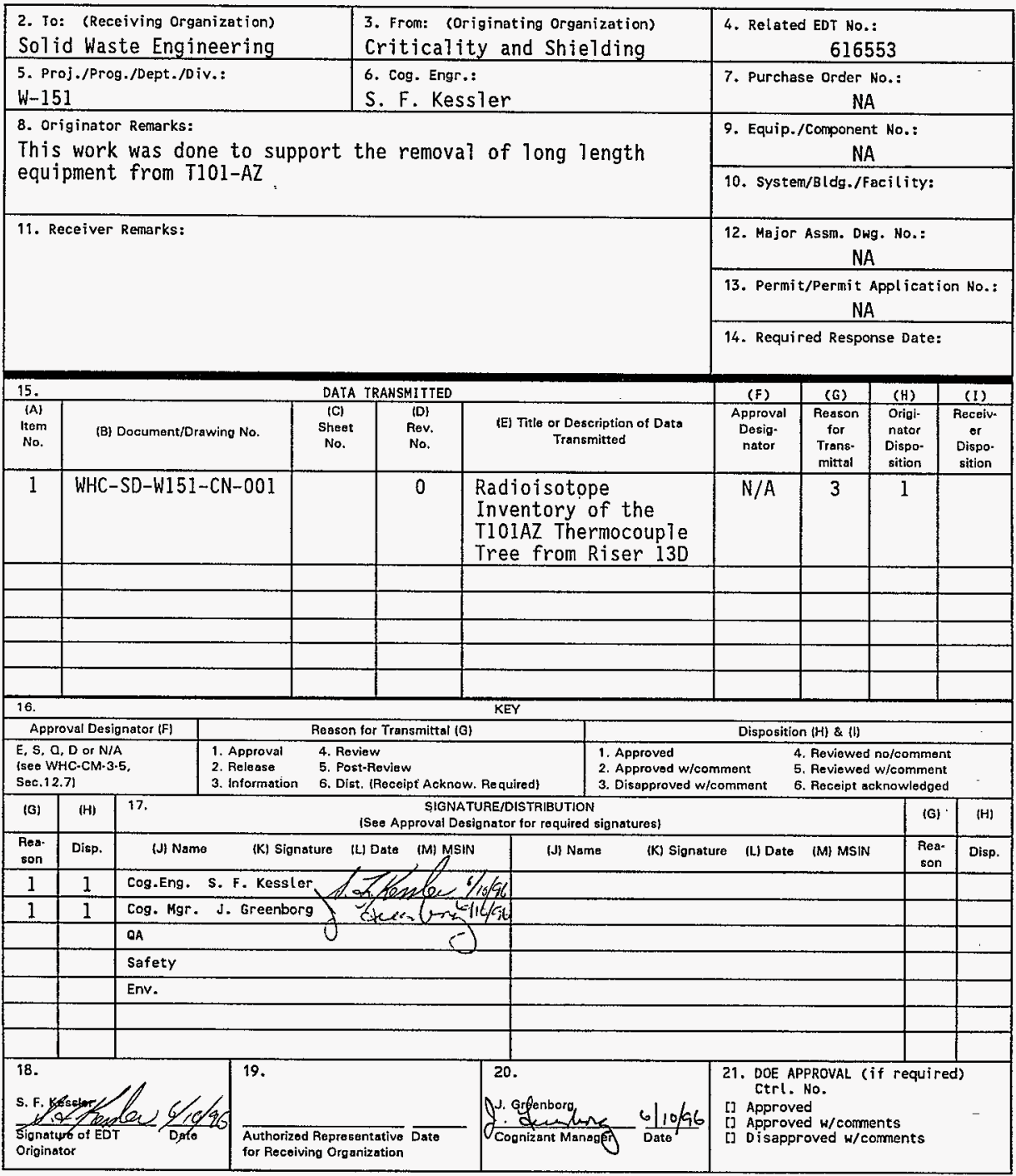




\section{Radioisotope Inventory of the T101AZ Thermocouple Tree from Riser 13D}

S. F. Kessier

Westinghouse Hanford Company, Richland, WA 99352

U.S. Department of Energy Contract DE-AC06-87RL10930

$\begin{array}{lll}\text { EDT/ECN: } & 616553 & \text { UC: } 510 \\ \text { Org Code: } & 8 M 730 & \text { Charge Code: } \\ \text { B\&R Code: } & \text { EW3130010 } & \text { Tota7 Pages: } 30\end{array}$

Key Words: Thermocouple tree, T-101AZ, Long Length Contaminated Equipment, LLCE

Abstract: The radionuclide inventory for the thermocouple tree removed from tank T101-AZ riser 130 was estimated using measured ${ }^{137} \mathrm{Cs}$ activity. This activity was measured by detectors as the tree was removed from the tank. Other radionuclide activities were estimated using the results of tank samples.

TRADEMARK DISCLAIMER. Reference herein to any specific comercial product, process, or service by trade name, trademark, manufacturer, or otherwise, does not necessarily constitute or imply its endorsement, recommendation, or favoring by the United States Government or any agency thereof or its contractors or subcontractors.

Printed in the United States of America. To obtain copies of this document, contact: WHC/BCS Document Control Services, P.O. Box 1970, Mailstop H6-08, Richland WA 99352, Phone (509) 372-2420; Fax (509) 376-4989.
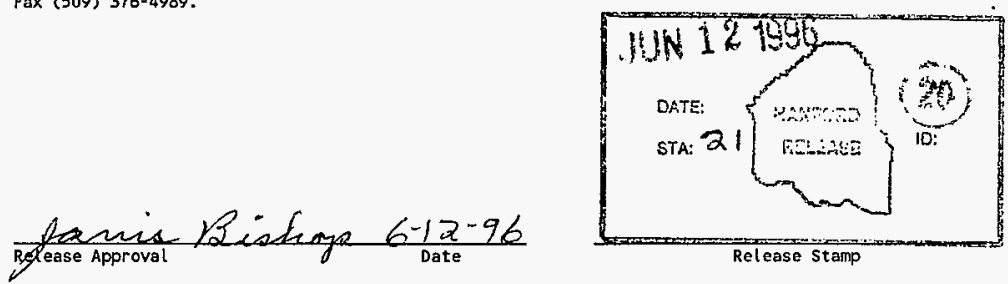

\section{Approved for Public Release}




\section{CHECKLIST FOR INDEPENDENT TECHNICAL REVIEW}

\section{DOCUMENT REVIEWED WHC-SD-W151-CN-001 RADIOISOTOPE ACTIVITY FOR THE T101AZ THERMOCOUPLE TREE REMOVED FROM RISER 13D AUTHOR(s) S. F. Kessler}

I. Method(s) of Review

$(\checkmark)$ Input data checked for accuracy

$(v)$ Independent calculation performed $(\checkmark)$ Hand calculation ( ) Alternate computer code:

( ) Comparison to experiment or previous results

() Alternate method (define)

II. Checklist (either check or enter NA if not applied)

$(\checkmark)$ Task completely defined

$(\checkmark)$ Activity consistent with task specification

$(v)$ Necessary assumptions explicitly stated and supported

$(\checkmark)$ Resources properly identified and referenced

$(\checkmark)$ Resource documentation appropriate for this application

$(\checkmark)$ Input data explicitly stated

( $)$ Input data verified to be consistent with original source

(WIA) Geometric model adequate representation of actual geometry

(W/A) Material properties appropriate and reasonable

$(N / A)$ Mathematical derivations checked including dimensional consistency

(-) Hand calculations checked for errors

(v) Assumptions explicitiy stated and justified

(v) Computer software appropriate for task and used within range of validity

(NIA) Use of resource outside range of established validity is justified

(WIA) Software runstreams correct and consistent with results

$(\sim)$ Software output consistent with input

(W/A) Results consistent with applicable previous experimental or analytical findings

$(\checkmark)$ Results and conclusions address a 11 points and are consistent with task requirements and/or estabiished limits or criteria

WA) Conclusions consistent with analytical results and established limits

$(N / A)$ Uncertainty assesment appropriate and reasonable () other (define)

III. Comments:

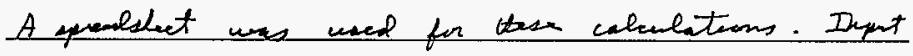

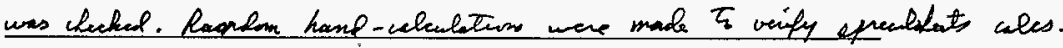

IV. REVIEWER:RF. Ruchiol DATE: Tume 3,1946 
WHC-SD-W151-CN-001, Rev. 0

Page 2 of 29

RADIOISOTOPE INVENTORY OF THE TIO1AZ THERMOCOUPLE TREE FROM RISER 13D

\subsection{PURPOSE}

The purpose of this calculation is to estimate the radionuclide inventory on the profile thermocouple tree removed from riser 130 on T101AZ. These data are used by Solid Waste Engineering to classify the thermocouple tree for disposal.

\subsection{METHODOLOGY}

Data are collected by the Flexible Receiver Detection System (FRRDS) as the thermocouple tree is removed from the tank and transmitted to "Criticality and Shielding".

Crane position and ${ }^{137} \mathrm{Cs}$ contamination levels are input to a WINGZ ${ }^{\mathrm{M}}$ spreadsheet to correct for geometry, calibration, create graphs for all three detectors and to calculate the inventory of the isotopes on the thermocouple tree. Results of tank waste samples have been used to calculate the ratio of each isotope with respect to ${ }^{137} \mathrm{Cs}^{1}$ for the liquid phase and sludge phase of material in the tank. A set of average ratios has also been calculated and are used to compare to the sludge and liquid phases.

Because the thermocouple tree is washed as it is removed to lower contamination levels, it may partially reinserted and removed several times for rewashing. When this happens, the activity recorded after the final wash is used to calculate the inventory.

The data are also averaged over al1 operable detectors.

\subsection{ASSUMPTIONS}

3.1 Because ${ }^{137} \mathrm{Cs}$ is soluble in water, it may be detected along the length of the thermocouple tree that has been in both the liquid or the sludge.

3.2 The liquid waste is not stratified and the ratio's of radionuclides to ${ }^{137} \mathrm{Cs}$ is constant.

3.3 The radionuclide distribution in the saltcake buildup on spacers or between profile thermocouple tubes is identical to that of the liquid.

3.4 The counting intervals of 1 minute represent $30.48 \mathrm{~cm}$ (1ft) of thermocouple tree movement.

\subsection{INPUT DATA}

Input data are provided by Radioanalytical chemistry ${ }^{2}$ as shown in Table 4.1.

"Wingz is a trademark of Informix Software, Inc. 
WHC-SD-W151-CN-001, Rev, 0

Page 3 of 29

\section{TABLE 4.1: DETECTOR INPUT DATA}

\begin{tabular}{|c|c|c|c|c|c|c|}
\hline \multicolumn{7}{|l|}{ Detector A1 } \\
\hline Coun & i Start Count & Cra & & & & \\
\hline Spectrum D & ate Time Time & Da & ite Time Pasition & Nuelide & Activity & Error \\
\hline A1650807360 & $5089607: 36: 17$ & 60. & 8-MAY-1996 7:37:17 & $7 \quad-833$ & & \\
\hline & & & $56 \mathrm{Cs} 137$ & $-3.798 E-02$ & $77.3 \%$ & \\
\hline A 1650807370 & $50896 \quad 07: 37: 24$ & 60. & 8-MAY-1996 $7: 38: 26$ & $6 \quad-1954$. & & \\
\hline & & & $55 \mathrm{CS} 137-$ & $-1.149 E-01$ & $22.6 \%$ & \\
\hline A1650807380 & $5089607: 38: 32$ & 60. & B-MAY-1996 $7: 39: 32$ & $2 \quad .3031$. & & \\
\hline & & & $27 \mathrm{CO} \quad 57$ & $2.316 E-02$ & $72.2 \%$ & \\
\hline & & & 55Cs $137-$ & $-6.836 E-02$ & $73.5 \%$ & \\
\hline A1650807390 & $50896 \quad 07: 39: 34$ & 60 & 8-MAY-1996 7:40:35 & $5 \quad-4057$. & & \\
\hline & & & & & $45.5 \%$ & \\
\hline A1650807400 & $50896 \quad 07: 40: 37$ & 60. & $\begin{array}{r}\text { 8-MAY-1996 7:41:38 } \\
55 C 5 \quad 137=\end{array}$ & - & $19.7 \%$ & \\
\hline A. 1650807420 & $5089607: 42: 00$ & 60. & B-MAY-1996 7:43:02 & .6087 & & \\
\hline & & & 11 NA $22-2$ & $-2.495 E-03$ & $546.7 \%$ & \\
\hline & & & $55 \mathrm{Cs} 137$ & $-1.077 E=01$ & $26.5 \%$ & \\
\hline & & & & $-7.025 \mathrm{E}-03$ & $546.7 \%$ & \\
\hline A1650807440 & 50896 07:44:15 & 60. & $\begin{array}{c}\text { 8-MAY-1996 } 7: 45: 15 \\
11 N A \quad 22-1\end{array}$ & $5 \quad-6087$. & & \\
\hline & & & $\begin{array}{lrl}11 \text { NA } & 22 & -1 \\
63 E U & 154 & -2\end{array}$ & $\begin{array}{l}-1.016 \mathrm{E}-02 \\
-2.860 \mathrm{E}-02\end{array}$ & $\begin{array}{l}132.0 \% \\
132.0 \%\end{array}$ & \\
\hline A1650807450 & $5089607: 45: 22$ & 60. & 8-MAY-1996 7:46:22 & $2 \quad-6087$ & & \\
\hline & & & $\begin{array}{l}\text { 45RHM } 105 \\
55 \mathrm{CS} \quad 137\end{array}$ & $\begin{array}{l}2.457 E-01 \\
-8.667 E-02\end{array}$ & $\begin{array}{l}29.5 \% \\
35.6 \%\end{array}$ & \\
\hline A1650808000 & $5089608: 00: 33$ & 60. & 8-MAY-1996 8:01:34 & $4 \quad-7817$ & & \\
\hline & & & $55 \mathrm{CS} 137$ & $-1.070 E-01$ & $23.8 \%$ & \\
\hline & & & 82PB 210 & $1.908 \mathrm{E}-04$ & $50.5 \%$ & \\
\hline A1650808010 & $5089608: 01: 40$ & 60. & 8-MAY-1996 8:02:43 & 3.7817 & & \\
\hline & & & $55 \mathrm{CS} 137=$ & $-1,286 E-01$ & $20.2 \%$ & \\
\hline A.1650808020 & $5089608: 02: 48$ & 60. & 8-MAY-1996 8:03:49 & $9 \quad-7817$. & & \\
\hline A1650808140 & $5089608: 14: 32$ & 60. & $\begin{array}{r}55 C S \quad 137- \\
\text { B-MAY-1996 } 8: 15 ; 34\end{array}$ & $\begin{aligned}-1.115 E \cdot 01 \\
4 \quad .10940\end{aligned}$ & $28.7 \%$ & \\
\hline & & & $\begin{array}{ll}55 \mathrm{CS} & 137 \\
54 \times \mathrm{XE} & 139\end{array}$ & $\begin{array}{l}1.148 E+02 \\
4.980 E-01\end{array}$ & $2 \begin{array}{c}0.5 \% \\
26.6 \%\end{array}$ & \\
\hline A1650808150 & $5089608: 15: 41$ & 60. & 8-MAY-1996 8:16:43 & $3 \quad 11187$. & & \\
\hline & & & $55 \mathrm{CS} \quad 137$ & $2.112 E+01$ & $1.4 \%$ & \\
\hline A1650808160 & $5089608: 16: 49$ & 60. & 8-MAY-1996 8:17:49 & $9 \quad-11187$. & & \\
\hline & & & 11 NA 22 & $3.038 E-02$ & $48.1 \%$ & \\
\hline & & & $\begin{array}{ll}\text { 55CS } & 137 \\
\text { 63EU } & 154\end{array}$ & $\begin{array}{l}2.153 \mathrm{E}+01 \\
8.554 \mathrm{E}-02\end{array}$ & $\begin{array}{r}1.6 \% \\
48.1 \%\end{array}$ & \\
\hline A1650808170 & $5089608: 17: 57$. & 60. & $\begin{array}{c}\text { 8-MAY-1996 } 8: 18: 58 \\
11 \text { NA } 22\end{array}$ & $\begin{array}{l}8-11187 . \\
2.137 E-02\end{array}$ & $103.2 \%$ & \\
\hline & & & $55 \operatorname{Cs} 137$ & $2.127 E+01$ & $1 \quad 1.6 \%$ & \\
\hline & & & 63EU 154 & $6.017 \mathrm{E}-02$ & $103.2 \%$ & \\
\hline A1650808190 & $5089608: 19: 05$ & 60. & $\begin{array}{c}\text { 8-MAY-1996 } 8: 20: 07 \\
\text { 55CS } 137\end{array}$ & 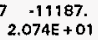 & $2.2 \%$ & \\
\hline A1650808200 & $5089608: 20: 12$ & 60. & 8-MAY-1996 B:21:13 & $3 \quad-11187$ & & \\
\hline & & & 55CS 137 & $2.132 E+01$ & $1.6 \%$ & \\
\hline A1650808210 & $5089608: 21: 21$ & 60. & 8-MAY-1996 8:22:22 & $2-11187$. & & \\
\hline & & & 11NA 22 & 3.245E-04 4 & $4905.1 \%$ & \\
\hline & & & $\begin{array}{ll}55 C S & 137 \\
63 E U & 154\end{array}$ & $\begin{array}{l}2.223 E+01 \\
9.138 E-044\end{array}$ & $\begin{array}{r}1.1 \% \\
4905.1 \%\end{array}$ & \\
\hline A1650808220 & $5089608: 22: 25$ & 60. & $\begin{array}{c}\text { 8-MAY-1996 } 8: 23: 28 \\
\text { 55CS } 137\end{array}$ & $\begin{array}{l}8 \quad-11187 . \\
2.139 E+01\end{array}$ & $1.8 \%$ & \\
\hline A1650808240 & $5089608: 24: 35$ & 60. & 8-MAY=1996 $8: 25: 36$ & $6 \quad-11187$ & $1.1 \%$ & \\
\hline & & & $55 \mathrm{Cs} 137$ & $2.129 E+01$ & $1.2 \%$ & \\
\hline A1650808250 & 50896 08:25:40 & 60. & $\begin{array}{c}\text { 8-MAY-1996 8:26:42 } \\
55 \text { CS } 137\end{array}$ & 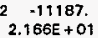 & $1.2 \%$ & \\
\hline A1650808260 & $5089608: 26: 45$ & 60. & $\begin{array}{r}\text { 8-MAY-1996 } 8: 27: 46 \\
40 Z R \quad 95 \quad 2\end{array}$ & $\begin{array}{l}-111187 \\
2.549 E-02\end{array}$ & $67.5 \%$ & \\
\hline & & & $55 \mathrm{cs} \quad 137$ & $2.198 E+01$ & $11.9 \%$ & \\
\hline 3300 & 896 OB:30:51 & 60. & 8-MAY-1996 8:31:52 & -11930 & & \\
\hline
\end{tabular}


A1650808310 50896 08:31:58

A1650808330 50896 08:33:06

A1650808340 50996 08:34:08

A1650808350 50896 08:35:10

A $1650808360 \quad 5089608: 36: 33$

At $650808370 \quad 50896$ 08:37:40

A1650808380 50896 08:38:41

A1650808410 50896 08:41:22

A 165080842050896 08:42:30

A $1650808430 \quad 50896$ 08:43:36

A 1650808440 50896 08:44:45

A1650808450 50896 08:45;54

A1650808470 50896 08:47:00

A $1650808480 \quad 50896$ 08:48:07

A $16508085005089608: 50: 22$

A 1650808510 50B96 08:51:30

A1650808520 50896 08:52:37
11NA $22 \quad 6.467 \mathrm{E}-03 \quad 217.3 \%$

$\begin{array}{llll}55 \mathrm{CS} & 137 & 2,593 \mathrm{E}+01 \quad 1.9 \%\end{array}$ G3EU $154 \quad 1.821 E-02 \quad 217.3 \%$

60. 8.MAY-1996 8:32:58 -13011 53) $128 \quad 9.377 E-01 \quad 25.4 \%$ 55Cs $137 \quad 4.471 E+01 \quad 0.9 \%$ 90THTH 232 3.043E + $01 \quad 21.1 \%$

60. 8-MAY-1996 8:34:07 -14135. 11NA $22 \quad 4.708 \mathrm{E}-02 \quad 42.1 \%$ $\begin{array}{lll}55 C S \quad 137 & 3.724 E+01 \quad 0.9 \%\end{array}$ G3EU $164 \quad 1.326 \mathrm{E}-01 \quad 42.1 \%$

60. 8-MAY-1996 8:35:10 -16162, 1INA 22 2.057E-02 $82.3 \%$ $\begin{array}{llll}\text { 56CS } & 137 & 1.558 \mathrm{E}+01 \quad 1.3 \%\end{array}$ $\begin{array}{llll}63 E U & 154 & 5.791 E .02 \quad 82.3 \%\end{array}$

60. 8-MAY-1996 8:36:10 -16141. 11NA $22+3.367 E-03 \quad 443.5 \%$ 55CS $137 \quad 9.172 E+00 \quad 1.8 \%$ G3EU $154 \cdot 9.481 \mathrm{E} \cdot 03 \quad 443.5 \%$ 69TM $170 \quad 3.023 E+\infty 0 \quad 27.9 \%$ 90TH $228 \quad 8.065 E+00 \quad 27.9 \%$ 9OTHTH $232 \quad 8.065 \mathrm{O}+00 \quad 27.9 \%$

60. 8-MAY-1996 8:37:34 -17509. $55 \mathrm{CS} \quad 137 \quad 3.529 \mathrm{E}+01 \quad 1.2 \%$

60. 8-MAY-1996 8:38:40 -17784. $\begin{array}{llll}55 \mathrm{CS} \quad 137 \quad 5.608 \mathrm{E}+01 \quad 1.0 \% & 0 .\end{array}$

60. 8-MAY-1996 8:39:43 -17784. $\begin{array}{llll}\text { 1INA } 22 & 1.464 E-02 & 104.3 \%\end{array}$ $\begin{array}{llll}27 C O & 60 & 1.830 E-02 & 49.9 \%\end{array}$ $\begin{array}{lll}55 C S \quad 137 & 5.398 E+01 \quad 1.0 \%\end{array}$ G3EU $154 \quad 4.121 \mathrm{E} .02 \quad 104.3 \%$

60. O-MAY-1996 8:42:22 -18876, 55CS $137 \quad 4.973 E+01 \quad 0.8 \%$

60. 8-MAY-1996 8:43:31 -19998. $\begin{array}{llll}55 \mathrm{CS} & 137 & 4.928 \mathrm{O}+01 & 0.8 \%\end{array}$ 9OTHTH $232 \quad 2.595 E+01 \quad 16.2 \%$

60. B-MAY-1996 8:44:37 -21082. 55CS $137 \quad 6.820 E+01 \quad 0.7 \%$

60. B-MAY-1996 8:45:46 -22203.

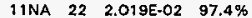
$\begin{array}{llll}55 C S \quad 137 & 9.692 \mathrm{E}+01 \quad 0.6 \%\end{array}$ 63EU $154 \quad 5.685 \mathrm{E}-02 \quad 97.4 \%$

60. 8-MAY-1996 8:46:55 -23333.

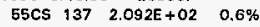

60. 8.MAY-1996 8:48:01 -24405, 28NI $65 \quad$ 5.297E-01 $42.9 \%$ $\begin{array}{lll}55 \mathrm{CS} & 137 \quad 7.847 \mathrm{E}+02 \quad 0.7 \%\end{array}$ 55CS $138 \quad 2.018 \mathrm{E} .01 \quad 51.5 \%$

60. B.MAY-1996 8:49:07 $\quad-25489$

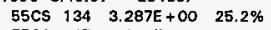
$\begin{array}{llll}55 \mathrm{CS} & 137 & 1.820 E+03 \quad 1.0 \%\end{array}$ 69TM $170 \quad 3.811 \mathrm{E}+02 \quad 6.4 \%$ 9O7H $228 \quad 1.017 \mathrm{E}+03 \quad 6.4 \%$ 90THTH $232 \quad 1,017 E+03 \quad 6.4 \%$

60. 8-MAY-1996 8:51:23 $\quad-27700$. $\begin{array}{llll}55 C 5 & 137 & 2.059 E+03 & 1.8 \%\end{array}$ $90 T H T H 232 \quad 1.291 \mathrm{E}+03 \quad 8.6 \%$

60. 8.MAY-1996 8:52:30 -28795. 55CS $137 \quad 1.189 E+03 \quad 0.5 \%$

60. B-MAY-1996 8:53:39 -29924 I3AL $28 \quad 2.249 \mathrm{E}-01 \quad 60.3 \%$ $\begin{array}{lll}531 & 134 \quad t .972 E+00 \quad 80.5 \%\end{array}$ $\begin{array}{llll}55 \mathrm{CS} & 137 & 1.442 \mathrm{E}+03 & 0.8 \%\end{array}$ 69TM $170 \quad 3.357 E+02 \quad 6.4 \%$ 90TH $228 \quad 8.955 \mathrm{E}+02 \quad 6.4 \%$ 9OTHTH232 B.955E + $02 \quad 6.4 \%$ 
WHC-SD-W151-CN-001, Rev. 0

Page 5 of 29

A1650808530 $5089608: 53: 43$

A1650808580 50896 08:58:03

A1650808590 50896 08:59:19

A1650809000 50896 09:00:26

A1650809110 50896 09:11:47

A $1650809120 \quad 5089609: 12: 56$

A1650809140 50896 09:14:04

A1650809150 50896 09:J6:11
60. 8-MAY-1996 8:54:45 -30998.

11 NA $22 \quad 6.501 E+00 \quad 21.8 \%$

$55 \mathrm{CS} \quad 137 \quad 2.005 E+03 \quad 1.1 \%$

63EU 154 1.837E+ O1 $21.8 \%$

69TM $170 \quad 6.887 E+02 \quad 7.5 \%$

90TH $228 \quad 1.837 E+03 \quad 7.5 \%$

9OTHTH232 1.837E + $03 \quad 7.5 \%$

60. 8-MAY.1996 8:59:03 $\quad-30030$

$27 \mathrm{Co} \quad 60 \quad 2.554 \mathrm{E}+00 \quad 48.2 \%$

$55 \mathrm{CS} \quad 137 \quad 1.883 \mathrm{E}+03 \quad 1.5 \%$

69TM $170 \quad 7.115 E+02 \quad 7.4 \%$

9OTH $228 \quad 1.898 E+03 \quad 7.4 \%$

9OTHTH232 $1.898 E+03 \quad 7.4 \%$

60. 8-MAY-1996 $9: 00: 21 \quad-29472$

$55 \mathrm{CS} \quad 137 \quad 1.559 E+03 \quad 0.7 \%$

90TH $228 \quad 1.096 \mathrm{E}+03 \quad 5.2 \%$

90THTH232 $1.096 \mathrm{E}+03 \quad 5.2 \%$

60, 6-MAY-1996 9:01:27 -30542.

$27 \mathrm{CO} \quad 56 \quad 1.692 \mathrm{E}+00 \quad 63.7 \%$

$41 \mathrm{NB} \quad 95 \quad 2.176 \mathrm{E}+00 \quad 56.1 \%$

51SB $125 \quad 1,302 \mathrm{E}+01 \quad 22.8 \%$

$55 \mathrm{CS} \quad 137 \quad 1.701 \mathrm{E}+03 \quad 1.0 \%$

69TM $170 \quad 5.728 E+02 \quad 6.0 \%$

$90 T H \quad 228 \quad 1.528 E+03 \quad 6.0 \%$

90THTH232 1.528E + $03 \quad 6.0 \%$

60. 8-MAY-1996 9:12:48 $\quad \mathbf{3 8 3 2 2}$.

$\begin{array}{lll}11 \mathrm{NA} & 22 & 5.491 \mathrm{E}+00 \quad 41.5 \%\end{array}$

$37 \mathrm{AB}$ B9 $7.007 \mathrm{E}+00 \quad 40.5 \%$

45AH $106 \quad 1.872 E+02 \quad 16.4 \%$

51SB $122 \quad 1.023 E+01 \quad 38.1 \%$

51SB $125 \quad 7.832 E+01 \quad 13.7 \%$

$55 C 5 \quad 137 \quad 1.674 E+03 \quad 1.8 \%$

63EU $154 \quad 1.546 E+01 \quad 41.5 \%$

69TM $170 \quad 1.003 E+03 \quad 11.5 \%$

90TH $228 \quad 2.676 \mathrm{E}+03 \quad 11.5 \%$

$90 T H T H 232 \quad 2.676 E+03 \quad 11.5 \%$

60. 8-MAY-1996 9:13:57 -39527

$\begin{array}{llll}11 \mathrm{NA} & 22 & 8.472 \mathrm{E}+00 \quad 14.5 \%\end{array}$

$27 \mathrm{CO} \quad 60 \quad 7.480 E+00 \quad 11.2 \%$

45RH $106 \quad 2.145 E+02 \quad 7.8 \%$

47AGM $108 \quad 8.967 E+00 \quad 21.0 \%$

$51 \mathrm{SB} \quad 125 \quad 6.854 \mathrm{E}+01 \quad 8.5 \%$

$55 \mathrm{CS} \quad 137 \quad 1.350 E+03 \quad 2.1 \%$

63EU $154 \quad 2.456 E+01 \quad 12.6 \%$

$69 \mathrm{TM} \quad 170 \quad 8.374 \mathrm{TE}+02 \quad 5.8 \%$

9OTH $228 \quad 2.218 \mathrm{E}+03 \quad 5.8 \%$

90THTH $232 \quad 2.218 E+03 \quad 5.8 \%$

60. 8-MAY-1996 9:15:06 $\quad-40737$

$11 \mathrm{NA} 22 \quad 6.746 \mathrm{E}+00 \quad 25.7 \%$

23V $52 \quad 3.027 E \div 00 \quad 56.2 \%$

$27 \mathrm{CO} \quad 57 \quad 7.951 E+00 \quad 22.5 \%$

$27 \mathrm{CO} \quad 60 \quad 4.364 E+00 \quad 59.9 \%$

36KRM $85 \quad 4.767 E+00 \quad 56.0 \%$

$39 Y \quad 91 \quad 2.478 E+03 \quad 33.0 \%$

45RH $106 \quad 1.952 E+02 \quad 14.0 \%$

51SB $125 \quad 8.264 E+01 \quad 10.8 \%$

$55 \mathrm{Cs} 137 \quad 1.709 \mathrm{E}+03 \quad 2.9 \%$

$\begin{array}{lll}\text { GSEU } 154 & 1.899 E+01 \quad 25.7 \%\end{array}$

69 TM $170 \quad 7.586 E+02 \quad 11.2 \%$

90TH $228 \quad 2.024 E+03 \quad 11.2 \%$

9OTHTH $232 \quad 2.024 E+03 \quad 11.2 \%$

60. 8-MAY+1996 9:16:12 -41889

23V $52 \quad 1.813 \mathrm{E}+00 \quad 112.4 \%$

$27 \mathrm{CO} \quad 60 \quad 2.649 \mathrm{E}+00 \quad 95.5 \%$

$30 Z N \quad 65 \quad 9.899 E+00 \quad 40.6 \%$

3asA $92 \quad 4.34 t E+00 \quad 58.8 \%$ 
WHC-SD-W151-CN-001, Rev. 0

Page 6 of 29

A1650809170 50896 09:17:15

A1650809190 50896 09:19:43

A1650809200 50896 09:20:52

A1650809210 50896 09:21:59

A1650809230 50896 09:23:05

A1650809240 50896 09:24:11
45RH $106 \quad 2.620 \mathrm{E}+02 \quad 16.5 \%$

$\begin{array}{llll}51 \mathrm{SB} & 125 & 7.470 \mathrm{E}+01 & 16.8 \%\end{array}$

$\begin{array}{lll}5 \mathrm{CS} & 137 & 1.609 \mathrm{E}+03 \quad 1.4 \%\end{array}$

59PAM $144 \quad 9.239 E+03 \quad 43.7 \%$

G9TM $170 \quad 9.687 E+02 \quad 9.2 \%$

90TH $228 \quad 2.584 \mathrm{E}+03 \quad 9.2 \%$

9OTHTH232 $2.584 \mathrm{E}+03 \quad 9.2 \%$

95AM $241 \quad 1.935 E+01 \quad 43.7 \%$

60. B-MAY-1996 9:18:15 -44038.

$12 M G \quad 27 \quad 1.102 E+01 \quad 52.2 \%$

$27 \mathrm{CO} \quad 60 \quad 4.880 E+00 \quad 48.3 \%$

40ZR $95 \quad 1.315 E+01 \quad 69.6 \%$

45RH $106 \quad 3.267 E+02 \quad 17.8 \%$

$\begin{array}{lllll}515 B & 125 & 5.066 \mathrm{E}+01 & 24.8 \%\end{array}$

$55 C 5134 \quad 1.452 E+01 \quad 56.2 \%$

55CS $137 \quad 1.237 E+03 \quad 1.7 \%$

69TM $170 \quad 1.145 \mathrm{E}+03 \quad 11.8 \%$

90TH $228 \quad 3.055 E+03 \quad 11.8 \%$

9OTHTH232 3.055E + $03 \quad 11.8 \%$

60. 8-MAY-1996 9:20:45 -43328.

11NA $22 \quad 5.669 \mathrm{E}+00 \quad 70.3 \%$

44RU $105 \quad 2.118 E+01 \quad 66.6 \%$

45RH $106 \quad 2.18 B E+02 \quad 18,1 \%$

4BCDM $115 \quad 3.004 E+02 \quad 77.5 \%$

$\begin{array}{llll}51 \mathrm{SB} & 125 \quad 6,921 \mathrm{E}+01 \quad 17.0 \%\end{array}$

$55 C S 134 \quad 9.286 E+00 \quad 55.5 \%$

$\begin{array}{llll}55 \mathrm{CS} & 137 & 1.231 \mathrm{E}+03 \quad 2.2 \%\end{array}$

63EU $154 \quad 1.596 E+01 \quad 70.3 \%$

69TM $170 \quad 9.804 E+02 \quad 8.1 \%$

$90 T 228 \quad 2.616 \mathrm{E}+03 \quad 8.1 \%$

9OTHTH 232 $2.616 E+03 \quad 8.1 \%$

94PU $240 \quad 2.577 \mathrm{E}+00 \quad 65.7 \%$

60. B-MAY-1996 9:21:54 -42154.

45RH $106 \quad 1.641 E+02 \quad 22,4 \%$ $54 X E \quad 135 \quad 4.273 E+00 \quad 68.9 \%$

$\begin{array}{llll}55 C S & 137 & 1.490 E+03 \quad 1.4 \%\end{array}$

G9TM $170 \quad 1.086 E+03 \quad 11.5 \%$ 90TH $228 \quad 2.897 \mathrm{E}+03 \quad 11.5 \%$

90THTH232 2.897E +03 $11.5 \%$

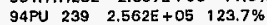

60. 8-MAY-1996 9:23:00 -42506.

45RH $106 \quad 2.159 E+02 \quad 17.4 \%$ $\begin{array}{llll}51 \mathrm{SB} & 125 & 5.926 \mathrm{E}+01 \quad 16.9 \%\end{array}$

$55 C \quad 137 \quad 1,473 E+03 \quad 2.8 \%$

5BCE $141 \quad 9.825 E+00 \quad 54.0 \%$

69TM $170 \quad 1.075 E+03 \quad 7.9 \%$

90TH $228 \quad 2.869 E+03 \quad 7.9 \%$

9OTHTH $232 \quad 2.869 \mathrm{E}+\mathrm{O} 3 \quad 7.9 \%$

60. 8-MAY-1996 9:24:06 -43663.

IBAR $41 \quad 1.518 E+01 \quad 168.9 \%$

$27 \mathrm{CO} \quad 60 \quad 5.886 \mathrm{E}+00 \quad 42.1 \%$

$40 Z R \quad 97 \quad 2.335 E+02 \quad 47.5 \%$

45RH $106 \quad 1.509 E+02 \quad 21.6 \%$

49INM $116 \quad 9.790 E+01 \quad 168.9 \%$

$515 B \quad 125 \quad 8.791 \mathrm{E}+01 \quad 11.6 \%$

$\begin{array}{llll}55 C S & 137 & 1.379 E+03 & 1.5 \%\end{array}$

64GD $153 \quad 2.053 E+01 \quad 54.8 \%$

69TM $170,1.279 E+03 \quad 13.0 \%$

9OTH $228 \quad 3.413 E+03 \quad 13.0 \%$

90THTH $232 \quad 3.413 \mathrm{E}+03 \quad 13.0 \%$

60. 8-MAY-1996 9:25:12 -44812 .

38SA $85 \quad 2.867 E-01 \quad 957.2 \%$

36KR $85 \quad 6.535 E+01 \quad 957.2 \%$

51SB $125 \quad 5.154 \mathrm{E}+01 \quad 20.1 \%$

$\begin{array}{llll}55 C S & 137 & 1.200 E+03 \quad 2.0 \%\end{array}$

69TM $170 \quad 1.170 \mathrm{E}+03 \quad 8.6 \%$ 
WHC-SD-W151-CN-001, Rev. 0

Page 7 of 29

A1650809280 $5089609: 28: 44$

A1650809290 50896 09:29:51

A1650809330 50896 09:33:15

A1650809440 50896 09:44:42

A1650810010 50896 10:01:03

A1650810020 50896 10:02:10

A1650810040 50896 10:04:22
90TH $228 \quad 3.122 E+03 \quad 8.6 \%$

90 THTH232 3.122E + $03 \quad 8.6 \%$

60. 8-MAY-1996 9:29:45 -43240 ,

$\begin{array}{llll}27 \mathrm{CO} & 60 & 7.852 \mathrm{E}+00 \quad 26.2 \%\end{array}$

42MO $101 \quad 1.623 E+01 \quad 148.8 \%$

45RH $106 \quad 2.575 E+02 \quad 11.9 \%$

$\begin{array}{llll}5158 & 125 & 7.237 \mathrm{E}+01 \quad 12.0 \%\end{array}$

52TEM $127 \quad 1.106 E+03 \quad 56.6 \%$

55CS $137 \quad 1.099 \mathrm{E}+03 \quad 1.8 \%$

57LA $140 \quad 2.189 E+00 \quad 61.8 \%$

69TM $170 \quad 9.040 E+02 \quad 11.9 \%$

$72 \mathrm{HF} \quad 175 \quad 6.531 \mathrm{E}+00 \quad 45.5 \%$

90TH $228 \quad 2.412 E+03 \quad 11.9 \%$

$92 \mathrm{U} \quad 232 \quad 2.813 E+03 \quad 56.6 \%$

90THTH232 $2.412 \mathrm{E}+03 \quad 11.9 \%$

60. 8-MAY-1996 9:30:52 $\quad-42277$

14S1 $31 \quad 9.785 E+03 \quad 44.5 \%$

$45 \mathrm{RH} \quad 106 \quad 2.434 \mathrm{E}+02 \quad 16.1 \%$

51SB $125 \quad 1.000 E+02 \quad 16.6 \%$

$55 \mathrm{CS} \quad 137 \quad 1.501 E+03 \quad 2.2 \%$

69TM $170 \quad 1.087 E+03 \quad 10.0 \%$

83BI $214 \quad 1.862 E+0168.8 \%$

$\begin{array}{ll}\text { BBRABI226 } & 1.830 E+01 \quad 68.8 \%\end{array}$

$90 \mathrm{TH} 228 \quad 2.900 \mathrm{E}+03 \quad 10.0 \%$

90THTH232 2.900E +03 10.0\%

60. B-MAY-1996 9:34:16 -44478,

$35 \times$ A5 $3,321 E+03 \quad 30,7 \%$

385R $85 \quad 1.451 E+01 \quad 30.7 \%$

45RH $106 \quad 1.653 E+02 \quad 23.3 \%$

$\begin{array}{llll}51 \mathrm{SB} & 125 & 8.174 \mathrm{E}+01 \quad 15.8 \%\end{array}$

55CS $137 \quad 1.266 \mathrm{E}+03 \quad 1.2 \%$

69TM $170 \quad 9.298 E+03 \quad 12.2 \%$

9OTH $228 \quad 3.462 \mathrm{E}+03 \quad 12.2 \%$

$\begin{array}{lll}\text { 9OTHTH232 } & 3.462 \mathrm{E}+03 & 12.2 \%\end{array}$

60. 8-MAY-1996 $9: 45: 43 \quad-44377$

3BSR $85 \quad 8.796 E+00 \quad 39.9 \%$

36KR $85 \quad 2.012 E+03 \quad 39.9 \%$

51SB 125 7.137E+ $01 \quad 17.3 \%$

531 $130 \quad 8.328 E+00 \quad 55.9 \%$

$55 \mathrm{CS} \quad 137 \quad 1.237 \mathrm{E}+03 \quad 1.6 \%$

56BA $140 \quad 3.440 E+01 \quad 55.9 \%$

69TM $170 \quad 1.232 \mathrm{E}+03 \quad 9.4 \%$

83B| $2127.229 E+01 \quad 91.6 \%$

$90 \mathrm{TH} \quad 228 \quad 3.266 \mathrm{E}+03 \quad 9.4 \%$

90THTH232 3.286E + $03 \quad 9.4 \%$

90THBL232 7.229E + $0191.6 \%$

60. B-MAY-1996 10:02:05 -54000.

$\begin{array}{lll}22 \mathrm{TI} & 51 \quad 7.066 \mathrm{E}+00 \quad 31.9 \%\end{array}$

37RB $86 \quad 4.132 E+01 \quad 49,3 \%$

45RH $105 \quad 3.249 E+01 \quad 31.9 \%$

45RH $106 \quad 9.863 E+0 t \quad 20.2 \%$

52TE $127 \quad 8.141 \mathrm{E}+02 \quad 31.7 \%$

$55 \mathrm{CS} \quad 137 \quad 9.391 \mathrm{E}+01 \quad 19.7 \%$

$69 \mathrm{TM} 170 \quad 1.157 \mathrm{E}+03 \quad 7.1 \%$

90TH $228 \quad 3.088 \mathrm{E}+03 \quad 7.1 \%$

90THTH232 $3.088 \mathrm{TE}+03 \quad 7.1 \%$

60. 8-MAY-1996 10:03:11 -55294.

$13 A L \quad 28 \quad 6.399 E+00 \quad 36.5 \%$

39YM $90 \quad 5.078 E+00 \quad 73.0 \%$

$\begin{array}{llll}51 \mathrm{SB} & 125 & 5.335 \mathrm{E}+01 \quad 30.9 \%\end{array}$

$55 \mathrm{CS} \quad 137 \quad 9.917 E+02 \quad 2.4 \%$

69TM $170 \quad 1.412 E+03 \quad 10.5 \%$

90TH $228 \quad 3.766 \mathrm{E}+03 \quad 10.5 \%$

90THTH232 $3.766 E+03 \quad 10.5 \%$

60. 8-MAY-1996 10:05:23 -57598

45RH $\quad 106 \quad 2.254 E+02 \quad 7.7 \%$ 
WHC-SD-W151-CN-001, Rev. 0

Page 8 of 29

A1650810050 50896 10:05:31

A1650810060 50896 10:06:39

A1650810070 $50896 \quad 10: 07: 49$

A1650810100 $5089610: 10 \div 05$

A1650810130 50896 10:13:28

A1650810140 50896 10:14:32

A1650810150 50896 10:15:36 $\begin{array}{lll}5158 & 125 & 4.250 E+01 \quad 13.7 \%\end{array}$ $55 \mathrm{CS} \quad 137 \quad 1.110 \mathrm{O}+03 \quad 1.9 \%$ 69TM $170 \quad 9.931 \mathrm{E}+02 \quad 5.2 \%$ 90TH $228 \quad 2.649 E+03 \quad 5.2 \%$ 90 THTH $232 \quad 2.649 E+03 \quad 5.2 \%$

60. 8-MAY-1996 10:06:33 57854.

45RH $106 \quad 7.756 \mathrm{E}+02 \quad 9.3 \%$ $51 \mathrm{SB} \quad 125 \quad 4.441 E+01 \quad 12,4 \%$ 53! $1313.592 E+00 \quad 56.2 \%$ 55CS $137 \quad 9.841 E+02 \quad 1.5 \%$ $54 X E \quad 139 \quad 7.816 E+00 \quad 55.6 \%$ 69TM $170 \quad 1.054 E+03 \quad 4.9 \%$ $90 \mathrm{TH} 228 \quad 2.811 \mathrm{E}+03 \quad 4.9 \%$ 90 THTH232 2.811E + 03 $4.9 \%$ 60. B-MAY-1996 10:07:39 -57854.

$11 \mathrm{NA} \quad 22 \quad 3,446 \mathrm{E}+00 \quad 25.3 \%$ 29CU $66 \quad 3.291 E+01 \quad 44.6 \%$

36KR $85 \quad 8.895 E+02 \quad 30.1 \%$ $38 S R \quad 85 \quad 3.88 B E+00 \quad 30.1 \%$ 45RH $106 \quad 1.624 \mathrm{E}+02 \quad 10.1 \%$

$51 \mathrm{SB} \quad 125 \quad 3.923 E+01 \quad 14.5 \%$ $55 C S \quad 137 \quad 9.183 E+02 \quad 1.5 \%$ $55 C 5 \quad 138 \quad 6.206 E-01 \quad 120.5 \%$

G3EU $154 \quad 1.006 E+01 \quad 24.2 \%$

69TM $170 \quad 1.004 E+03 \quad 4.6 \%$ SOTH $228 \quad 2.679 \mathrm{E}+03 \quad 4.6 \%$ SOTHTH $232 \quad 2.679 E+03 \quad 4.6 \%$ $91 \mathrm{PA} 234 \quad 3.074 \mathrm{E}+02 \quad 55.2 \%$

60. B-MAY-1996 10:08:51 .57854. $27 \mathrm{CO} \quad 60 \quad 2.562 \mathrm{E}+00 \quad 27.3 \%$ $45 R H \quad 106 \quad 1.796 E+02 \quad 9.3 \%$

$51 \mathrm{SB} \quad 125 \quad 3.753 \mathrm{E}+01 \quad 11.4 \%$ $55 \mathrm{CS} \quad 137 \quad 9.906 \mathrm{E}+02 \quad 2.0 \%$ 69TM $170 \quad 1.152 \mathrm{E}+03 \quad 6.4 \%$ 90 TH $228 \quad 3.073 E+03 \quad 6.4 \%$ 90THTH232 $3.073 \mathrm{E}+03 \quad 6.4 \%$

60. 8-MAY-1996 10:11:06 -56708.

$27 \mathrm{CO} \quad 60 \quad 2.717 \mathrm{E}+00 \quad 24.7 \%$ 45RH $106 \quad 1.909 E+02 \quad 10.0 \%$ $515 B \quad 125 \quad 4.611 E+01 \quad 11.9 \%$ $55 \mathrm{CS} \quad 137 \quad 9.9015+02 \quad 1,2 \%$ G9TM $170 \quad 9.446 E+02 \quad 4.5 \%$ 90TH $228 \quad 2.520 E+03 \quad 4.6 \%$ 9OTHTH $232 \quad 2.520 E+03 \quad 4.5 \%$

60. 8-MAY-1996 10:14:30 $\quad .56345$.

29CU $66 \quad 5.113 E+01 \quad 51.4 \%$ 45AH $106 \quad 1.710 \mathrm{E}+02 \quad 18.2 \%$ 51SB $125 \quad 2.526 \mathrm{E}+01 \quad 22.1 \%$ $55 \mathrm{CS} \quad 137 \quad 1.069 E+03 \quad 1.5 \%$ 69TM $170 \quad 1,111 \mathrm{E}+03 \quad 5.7 \%$ $90 T H \quad 228 \quad 2.965 E+03 \quad 5.7 \%$ 90THTH232 2.965E + $03 \quad 5.7 \%$

60. 8-MAY-1996 10:15:33 -57455. 4OZR $95 \quad 4.551 E+00 \quad 61.0 \%$ 45RH $106 \quad 2.195 \mathrm{E}+02 \quad 7.3 \%$ $51 \mathrm{SB} 125 \quad 3.717 \mathrm{E}+01 \quad 11.2 \%$ $55 \mathrm{CS} \quad 137 \quad 1.044 \mathrm{E}+03 \quad 2.1 \%$ 69TM $170 \quad$ 8.657E + $02 \quad 5.1 \%$ 90TH $228 \quad 2.310 E+03 \quad 5.1 \%$ 9OTHTH232 2.310E + O3 $5.1 \%$ 60. B.MAY-1996 10:16:36 -58547.

$\begin{array}{llll}11 \mathrm{NA} & 22 & 2.499 \mathrm{E}+00 \quad 23.4 \%\end{array}$ $27 C O \quad 60 \quad 2.231 E+\infty 0 \quad 23.5 \%$ 55CS $137 \quad 7.976 E+02 \quad 1.5 \%$ 63EU $154 \quad 7.038 E+00 \quad 23.4 \%$ 69TM $170 \quad 8,841 E+02 \quad 4.7 \%$ 
WHC-SD-W151-CN-001, Rev. 0

Page 9 of 29

90TH $228 \quad 2.359 E+03 \quad 4.7 \%$ 9OTHTH232 $2.359 E+03 \quad 4.7 \%$

A1650810170 50896 10:17:02 60. 8-MAY-1996 10:18:04 -60085.

47AGM $108 \quad 1.411 E+01 \quad 39.5 \%$

531 $135 \quad 1.822 E+01 \quad 63.4 \%$

69TM $170 \quad 1.621 \mathrm{E}+03 \quad 7.2 \%$

90TH $228 \quad 4.324 E+03 \quad 7.2 \%$

$90 \mathrm{THTH} 232 \quad 4.324 \mathrm{E}+03 \quad 7.2 \%$ 
WHC-SD-W151-CN-001, Rev. 0

Page 10 of 29

Detector A2

\begin{tabular}{|c|c|c|c|c|c|}
\hline A2650808450 & $5089608: 45: 54$ & 60. & 8-MAY-1996 8:46:55 & $5 \quad-23333$ & \\
\hline & & & $55 \mathrm{Cs} 137$ & $3.658 E+\infty 0$ & $35.6 \%$ \\
\hline A2650808470 & $5089608: 47: 00$ & 60. & 8-MAY-1996 8:48:01 & $1 \quad-24405$ & \\
\hline & & & 55Cs 137 & $1.847 E+01$ & $16.9 \%$ \\
\hline A2650808480 & $5089608: 48: 07$ & 60. & 8-MAY-1996 8:49:07 & -25489 & \\
\hline & & & 55CS 137 & $3.243 E+01$ & $12.6 \%$ \\
\hline A.2650808490 & $5089608: 49: 15$ & 60. & 8-MAY-1996 8:50:17 & $7 \quad-26627$ & \\
\hline & & & 5ISB 126 & $4.604 E+00$ & $29.8 \%$ \\
\hline & & & $55 \mathrm{CS} 137$ & $5.999 E+01$ & $9.5 \%$ \\
\hline A 2650808500 & $5089608: 50: 22$ & 60. & $\begin{array}{c}\text { B-MAY-1996 8:51:23 } \\
55 \mathrm{CS} 137\end{array}$ & $\begin{array}{c}-27700 \\
4.838 E+01\end{array}$ & $10.6 \%$ \\
\hline A2650808510 & 50896 08:51:30 & 60. & 8.MAY-1996 8:52:30 & -28795 & \\
\hline & & & $55 \mathrm{Cs} 137$ & $2.051 E+01$ & $16.6 \%$ \\
\hline A.2650808520 & $5089608: 52: 37$ & 60. & 8-MAY-1996 8:53:38 & .9. 29924 & \\
\hline & & & 55 Cs 137 & $3.170 \mathrm{E}+01$ & $12.8 \%$ \\
\hline A2650808530 & $5089608: 53: 43$ & 60. & 8-MAY-1996 B:54:45 & -30998 & \\
\hline & & & 11 NA 22 & $2.758 E+00$ & $18.1 \%$ \\
\hline & & & $27 \mathrm{co} 60$ & 6.68BE-01 & $45.5 \%$ \\
\hline & & & $55 \mathrm{CS} \quad 137$ & $5.504 E+01$ & $10.7 \%$ \\
\hline & & & 63EU 154 & $7.765 E+00$ & $18.1 \%$ \\
\hline A.2650808550 & $5089608: 55: 55$ & 60. & 8-MAY-1996 8:56:57 & $7 \quad-31930$ & \\
\hline & & & $11 \mathrm{NA} \quad 22$ & $5.087 E+01$ & $4.2 \%$ \\
\hline & & & $27 \mathrm{CO}$ & $2.360 E+01$ & $6.1 \%$ \\
\hline & & & $55 \mathrm{CS} 137$ & $1.732 \mathrm{E}+02$ & $9.3 \%$ \\
\hline & & & 57LA 140 & $3,188 E+00$ & $17.3 \%$ \\
\hline & & & G3EU 154 & $1.436 E+02$ & $4.1 \%$ \\
\hline A2650808570 & 50896 08:57:01 & 60. & 8.MAY-1996 8:58:03 & .30879 & \\
\hline & & & $11 \mathrm{NA} \quad 22$ & $2.082 E+01$ & $6.5 \%$ \\
\hline & & & $27 \mathrm{co} \quad 60$ & $6.566 E+00$ & $9.9 \%$ \\
\hline & & & $55 \operatorname{Cs~} 137$ & $8.820 E+01$ & $8.3 \%$ \\
\hline & & & $57 \mathrm{LA} \quad 140$ & 9.685E-01 & $\mathbf{3 2 . 8 \%}$ \\
\hline A2650808580 & 50896 08:58:06 & 60. & $\begin{array}{cc}\text { 63EU } & 154 \\
\text { 8-MAY-1996 } & \text { 0:59:06 }\end{array}$ & $\begin{array}{c}5.846 E+01 \\
16-30079\end{array}$ & $6.4 \%$ \\
\hline & & & $11 N A 22$ & $2.196 \mathrm{E}+00$ & $23.2 \%$ \\
\hline & & & $27 \mathrm{CO} \quad 60$ & $7.902 E-01$ & $40.3 \%$ \\
\hline & & & $55 \mathrm{CS} \quad 137$ & $5.276 E+01$ & $10.0 \%$ \\
\hline & & & G3EU 154 & $6.183 E+00$ & $23.2 \%$ \\
\hline A2650808590 & 50896 OB:59:19 & 60. & 8-MAY-1996 9:00:21 & $1 \quad-29472$ & \\
\hline & & & $55 \mathrm{CS} 137$ & $3.746 \mathrm{E}+01$ & $11.6 \%$ \\
\hline A2650809000 & $5089609: 00: 26$ & 60. & 8-MAY-1996 9:01:27 & $7 \quad-30542$ & \\
\hline & & & $11 \mathrm{NA} 22$ & $1.719 E+00$ & $25.1 \%$ \\
\hline & & & $55 \mathrm{cs} 137$ & $3.976 E+01$ & $11.9 \%$ \\
\hline & & & 63EU 154 & $4.841 E+00$ & $25.1 \%$ \\
\hline A 2650809010 & 50896 09:01:32 & 60. & 8-MAY-1996 9:02:33 & $13 \quad-31620$ & \\
\hline & & & $11 N A 22$ & $1.091 E+01$ & $8.8 \%$ \\
\hline & & & $27 \mathrm{co} \quad 60$ & $4.530 E+\infty 0$ & $12.1 \%$ \\
\hline & & & $55 \mathrm{CS} \quad 137$ & $8.148 E+01$ & $8.2 \%$ \\
\hline & & & 63EU 154 & $3.072 \mathrm{E}+01$ & $8.8 \%$ \\
\hline A2650809020 & 50896 09:02:40 & 60 . & 8-MAY-1996 9:03:42 & $2-32729$. & \\
\hline & & & 11 NA 22 & $4.871 E+01$ & $4.3 \%$ \\
\hline & & & $27 \mathrm{CO} \quad 60$ & $1.823 E+01$ & $5.5 \%$ \\
\hline & & & $55 \mathrm{CS} 137$ & $1.688 \mathrm{E}+02$ & $6.2 \%$ \\
\hline & & & 57LA 140 & $3.394 E+\infty 0$ & $25.0 \%$ \\
\hline & & & 63EU 154 & $1.350 E+02$ & $4.2 \%$ \\
\hline A 2650809030 & $5089609: 03: 49$ & 60 , & 8-MAY-1996 9;04:5 & i1 -33860 & \\
\hline . & & & 11NA 22 & $2.268 E+02$ & $2.5 \%$ \\
\hline & & & $27 \mathrm{CO} 60$ & $7.675 \mathrm{E}+01$ & $3.2 \%$ \\
\hline & & & $55 \mathrm{Cs} 137$ & $5.115 E+02$ & $4.6 \%$ \\
\hline & & & 57LA 140 & $1.379 \mathrm{gE}+01$ & $7.8 \%$ \\
\hline & & & 63EU 154 & $6.408 E+02$ & $2.4 \%$ \\
\hline A 2650809040 & $5089609: 04: 58$ & 60. & 8-MAY-1996 9:06:00 & $10 \quad-35029$ & \\
\hline & & & IINA 22 & $9.902 E+01$ & $4.0 \%$ \\
\hline & & & 270060 & $4.221 E+01$ & $4.6 \%$ \\
\hline & & & $55 \mathrm{CS} 137$ & $2.368 E+02$ & $6.8 \%$ \\
\hline
\end{tabular}


WHC-SD-W151-CN-001, Rev. 0 Page 11 of 29

\section{A2650809060 50896 09:06:07}

A2650809070 50896 09:07:15

A2850809080 50896 09:08:23

A2650809090 50896 09:09:30

A2650809100 50896 09:10:41

A2650809110 50896 09:11:47

A2650809120 50896 09:12:56

A2650809140 50896 09:14:04

A2650809150 50896 09:15:11

A2650809160 50896 09:16:20

A2650809170 50896 09:17:26

A2650809180 50896 09:18:36
57LA $140 \quad 5.281 E+00 \quad 14.6 \%$ G3EU $154 \quad 2.703 E+02 \quad 3.7 \%$ 60. 8-MAY-1996 9:07:09 $\quad .35299$.

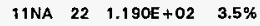
$\begin{array}{lllll}27 C O & 60 & 4.641 E+01 & 4.2 \%\end{array}$ $55 \mathrm{CS} \quad 137 \quad 2.264 \mathrm{E}+02 \quad 6.3 \%$ 57LA $140 \quad$ B.179E +00 $10.4 \%$ G3EU $154 \quad 3.372 \mathrm{E}+02 \quad 3.5 \%$

60. 8-MAY-1996 9:08:15 -35299.

$\begin{array}{llll}\text { I1NA } 22 & 1.237 \mathrm{E}+02 \quad 2.7 \%\end{array}$ $\begin{array}{llll}27 C O & 60 & 4.476 E+01 & 4.3 \%\end{array}$ 55CS $137 \quad 2.130 E+02 \quad 6.0 \%$ $\begin{array}{llll}\text { 57LA } & 140 \quad 9.014 E+00 \quad 12.5 \%\end{array}$ 63EU $154 \quad 3.495 E+02 \quad 2.7 \%$

60. B-MAY-1996 9:09:24 -35299.

$\begin{array}{llll}11 \mathrm{NA} & 22 & 1.132 \mathrm{E}+02 & 2.8 \%\end{array}$ $\begin{array}{llll}27 C 0 & 60 & 4.148 E+01 & 3.7 \%\end{array}$ $\begin{array}{llll}55 C S & 137 & 2.246 E+02 & 6.2 \%\end{array}$ 57LA $140 \quad 8.720 E+00 \quad 12.6 \%$ G3EU $154 \quad 3.198 E+02 \quad 2.8 \%$ 8OHG $197 \quad 8.680 \mathrm{E}+03 \quad 60.8 \%$

60. 8-MAY-1996 9:10:30 +35924

11NA $22 \quad 9.130 \mathrm{E}+01 \quad 3.2 \%$ $27 \mathrm{CO} \quad 60 \quad 2.947 \mathrm{E}+01 \quad 4.2 \%$ $55 \mathrm{CS} \quad 137 \quad 1.531 E+02 \quad 6.8 \%$ 57LA $140 \quad 6.584 E+00 \quad 11.3 \%$ 63EU $154 \quad 2.583 E+02 \quad 3.1 \%$

60, 8-MAY-1996 9:11:42 37168.

11NA $22 \quad 4.593 E+00 \quad 17.5 \%$ 215C $46 \quad 3.030 E-01 \quad 176.2 \%$ $27 C 0 \quad 60 \quad 2.971 E+00 \quad 16.9 \%$ G3EU $154 \quad 1.293 E+01 \quad 17.5 \%$

60. B.MAY-1996 9:12:48 $\quad-38322$. $11 \mathrm{NA} 22 \quad 1.852 \mathrm{E}+00 \quad 23.2 \%$ 55CS $137 \quad 3.956 E+01 \quad 11.6 \%$ 63EU $154 \quad 5.216 \mathrm{E}+00 \quad 23.2 \%$

60. 8-MAY-1996 9:13:57 -39527. I1NA $22 \quad 1.361 E+00 \quad 27.2 \%$ $27 \mathrm{CO} \quad 60 \quad 1.191 \mathrm{E}+00 \quad 22.1 \%$

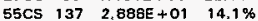
G3EU $164 \quad 3.831 E+00 \quad 27.2 \%$

60. 8.MAY-1996 9:15:06 -40737.

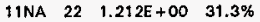
55CS $137 \quad 4.092 E+01 \quad 12.0 \%$ 63EU $154 \quad 3.411 E+\infty \quad 31.3 \%$

60. 8-MAY-1996 9:16:12 -418B9. I1NA $22 \quad 1.107 E+00 \quad 28.7 \%$ $27 \mathrm{CO} \quad 60 \quad 1.426 \mathrm{E}+00 \quad 19.5 \%$ $\begin{array}{llll}55 \mathrm{CS} & 137 & 3.244 \mathrm{E}+01 & 13.1 \%\end{array}$ G3EU $154 \quad 3.116 E+00 \quad 28.7 \%$

60. B-MAY-1996 9:17:21 -43095.

IINA $22 \quad 1.138 E+00 \quad 31.3 \%$ $\begin{array}{llll}27 C O & 60 & 1.018 E+\infty 0 & 25.5 \%\end{array}$ 55 CS $137 \quad 2.584 E+01 \quad 15.1 \%$ GBEU $154 \quad 3.205 E+00 \quad 31.3 \%$ 8OHG $197-3.503 E+03 \quad 210.3 \%$

60. 8-MAY-1996 9:18:27 .44246. $\begin{array}{llll}\text { IINA } 22 & 1.350 E+00 \quad 35.7 \%\end{array}$ $27 \mathrm{CO} \quad 60 \quad 1.329 \mathrm{E}+00 \quad 22.4 \%$ $46 \mathrm{PD} \quad 109 \quad$ 8.151E+04 $54.3 \%$ 63EU $154 \quad 3,802 E+\infty \quad 35.7 \%$

60. B-MAY-1996 9:19:36 .44495. 1INA $22 \quad 2.427 E+00 \quad 23.1 \%$ $27 \mathrm{CO} \quad 60 \quad 9.174 \mathrm{E}-01 \quad 24.9 \%$ 55CS $137 \quad 3.271 E+01 \quad 13.7 \%$ G3EU $154 \quad 6.834 E+00 \quad 23.1 \%$ 


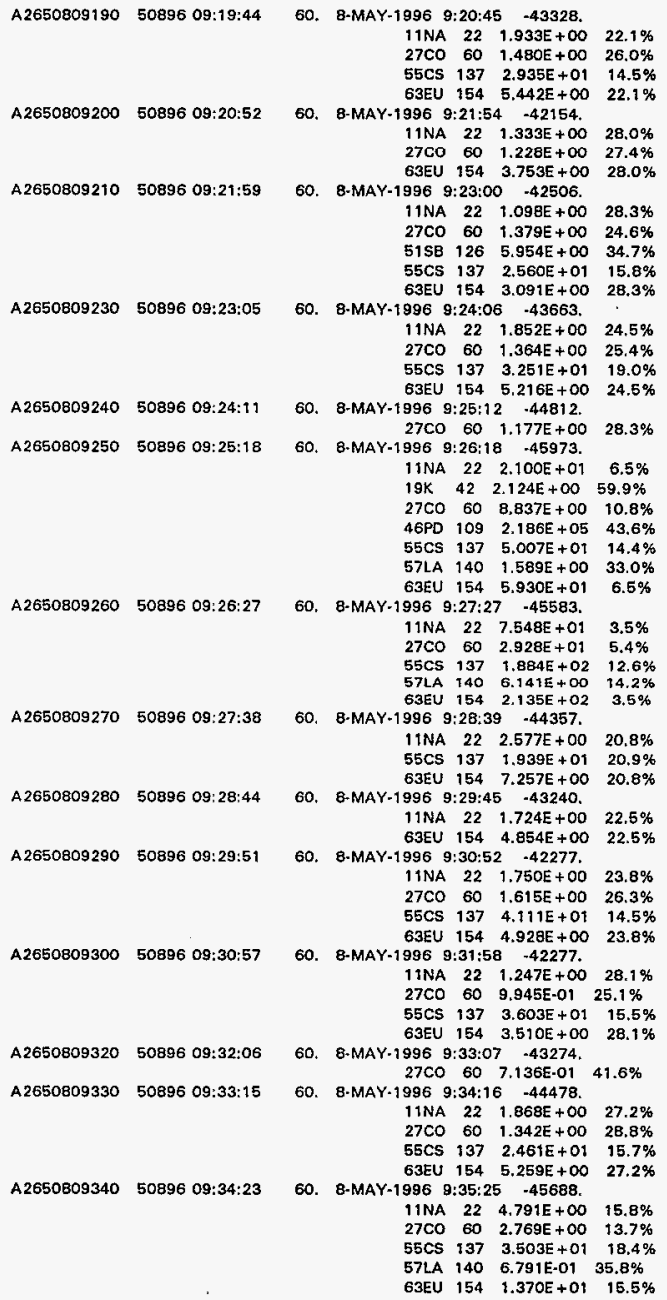


A2650809350 50896 09:35:32

A2650809360 $50896 \quad 09: 36: 41$

A2650809370 50896 09:37:49

A2650809380 50896 09:38:57

A2650809400 50896 09:40:03

A2650809410 50896 09:41:08

A2650809420 50896 09:42:24

A2650809430 50896 09:43:33

A2650809440 $5089609: 44: 42$

A2650809450 50896 09:45:51
60. 8-MAY-1996 9:36:34 -46596.

I1NA $22 \quad 8.537 E+01 \quad 3.3 \%$

IBAR 41 8.026E-01 $81.3 \%$

$27 \mathrm{CO}$ 60 $3.287 \mathrm{E}+016.6 \%$

49INM $116 \quad 9.461 E-01 \quad 81.3 \%$

$55 \mathrm{CS} \quad 137 \quad 1.919 \mathrm{E}+02 \quad 6.0 \%$

57LA $140 \quad 4.939 E+00 \quad 13.4 \%$

63EU $154 \quad 2.407 E+02 \quad 3.2 \%$

60. 8.MAY-1996 9:37:43 -46596.

$11 \mathrm{NA} 228.712 \mathrm{E}+01 \quad 3.1 \%$

$27 C 0$ 6O $2.696 E+01 \quad 5.7 \%$

$55 C 5137$ 1.460E + $02 \quad 6.8 \%$

5TLA $140 \quad 6.404 E+00 \quad 12.1 \%$

63EU $154 \quad 2.463 E+02 \quad 3.1 \%$

60. 8-MAY-1996 9:38:51 -46596

1 INA $22 \quad 8.519 E+01 \quad 3.3 \%$

$27 \mathrm{CO} \quad 60 \quad 3.116 \mathrm{E}+01 \quad 5.6 \%$

$55 \mathrm{Cs} 137 \quad 1.319 E+02 \quad 11.5 \%$

57LA $140 \quad 5,681 E+00 \quad 12.5 \%$

59PA $144 \quad 1.864 \mathrm{E}+02 \quad 63.4 \%$

G3EU $154 \quad 2.407 E+02 \quad 3.3 \%$

60. 8-MAY-1996 9:39:58 $\quad-47080$.

$11 \mathrm{NA} 22 \quad 7.512 \mathrm{E}+01 \quad 3.9 \%$

$27 \mathrm{CO} \quad 60 \quad 2.6 \mathrm{~B} 3 \mathrm{E}+01 \quad 5.5 \%$

55CS $137 \quad 1.350 \mathrm{E}+02 \quad 7.6 \%$

57LA $140 \quad 6.192 E+00 \quad 11.6 \%$

G3EU $154 \quad 2.115 E+02 \quad 3.9 \%$

60. 8-MAY-1996 9:41:04 -47100.

11NA $22 \quad 5.193 \mathrm{E}+02 \quad 1.4 \%$

$27 \mathrm{CO} \quad 60 \quad 1.771 \mathrm{E}+02 \quad 2.4 \%$

$29 \mathrm{CU} 64 \quad 5.174 \mathrm{E}+02 \quad 49.3 \%$

55CS $137 \quad 8.002 E+02 \quad 3.5 \%$

S7LA $140 \quad 3.564 E+01 \quad 5.8 \%$

63EU $154 \quad 1.462 \mathrm{E}+03 \quad 1.4 \%$

60. 8-MAY-1996 $9: 42: 10 \quad-47138$.

$11 \mathrm{NA} \quad 22 \quad 5.452 \mathrm{E}+02 \quad 1.4 \%$

$27 \mathrm{CO} \quad 60 \quad 1.849 \mathrm{E}+02 \quad 3.4 \%$

$55 \mathrm{CS} \quad 137 \quad 1.047 \mathrm{E}+03 \quad 4.6 \%$

57LA $140 \quad 3.993 E+01 \quad 5.0 \%$ 63EU $154 \quad 1.535 E+03 \quad 1.4 \%$

60. 8-MAY-1996 9:43:25 -45927.

$80 \quad 19 \quad 2.161 E+00 \quad 66.3 \%$

$11 \mathrm{NA} 22 \quad 8.803 \mathrm{E}+01 \quad 4.0 \%$

$27 C 0 \quad 60 \quad 3.383 E+01 \quad 5.1 \%$

46PO $109 \quad 6.899 E+04 \quad 85.2 \%$

$55 \mathrm{CS} 137 \quad 1.762 \mathrm{E}+02 \quad 6.9 \%$

57LA $140 \quad 7.278 E+00 \quad 11.3 \%$

63EU $154 \quad 2.479 E+02 \quad 4.0 \%$

60. 8-MAY-1996 9:44:34 -44750 .

$11 \mathrm{NA} \quad 22 \quad 9.634 \mathrm{E}+\infty \quad 10.1 \%$

$27 \mathrm{CO} \quad 60 \quad 3.265 E+\infty \quad 14.9 \%$

$55 \mathrm{CS} 137 \quad 4.866 \mathrm{E}+01 \quad 11.6 \%$

57LA $140 \quad 9.545 E-01 \quad 32.6 \%$

63EU $154 \quad 2.734 E+01 \quad 9.9 \%$

60. 8-MAY-1996 9:45:43 $\quad-44377$.

$11 \mathrm{NA} \quad 22 \quad 1.569 \mathrm{E}+00 \quad 23.4 \%$ $27 \mathrm{CO} \quad 60 \quad 1.284 \mathrm{E}+00 \quad 22.1 \%$

55CS $137 \quad 2.443 E+01 \quad 16.7 \%$

63EU $154 \quad 4.418 E+00 \quad 23.4 \%$

60. B-MAY-1996 $9: 46: 53 \quad-45603$

11NA $22 \quad 4,698 E+00 \quad 15.9 \%$

18AR $415.069 E-01 \quad 52.9 \%$

$27 C 0 \quad 60 \quad 2,240 E+00 \quad 19.7 \%$

$491 \mathrm{NM} 116 \quad 5.975 \mathrm{E}-01 \quad 52.9 \%$

$55 \mathrm{CS} 137 \quad 2.853 \mathrm{E}+01 \quad 15.6 \%$

57LA $140 \quad 3.667 \mathrm{E}-01 \quad 41.4 \%$ 
WHC-SD-W151-CN-001, Rev. 0

Page 14 of 29

A2650809460 50896 09:46:58

A2650803490 50856 09:49:13

A2650809500 50896 09:50:18

A2650809510 50896 09:51:57

A2650809530 50896 09:53:07

A2650809540 50896 09:54:16

A2650B09550 50896 09:55:22

A2650809560 50896 09:56:31

A2650809570 50896 09:57:38

A2650809580 $50896 \quad 09: 58: 46$

A2650809590 $5089609: 59: 55$
63EU $154 \quad 1.336 E+01 \quad 15.4 \%$

60. 8-MAY-1996 9:47:59 -46723.

11NA $22 \quad 8.817 \mathrm{E}+01 \quad 4.2 \%$

$27 C 0 \quad 60 \quad 3.040 E+01 \quad 4.5 \%$

$55 \mathrm{CS} 137 \quad 1.769 \mathrm{E}+02 \quad 6.8 \%$

57LA $140 \quad 5.369 E+00 \quad 12.9 \%$

G3EU $154 \quad 2.491 E+02 \quad 4.1 \%$

60. 8-MAY-1996 9:50:14 -48177

$11 \mathrm{NA} 22 \quad 4.822 \mathrm{E}+02 \quad 1.5 \%$

$2700 \quad 60 \quad 1.574 E+02 \quad 2.8 \%$

$55 \mathrm{CS} \quad 137 \quad 7.767 \mathrm{E}+02 \quad 3.5 \%$

57LA $140 \quad 3.405 E+01 \quad 6.4 \%$

63EU $154,1.358 E+03,1.5 \%$

60. 8-MAY-1996 $9: 51: 20 \quad-49323$

$11 \mathrm{NA} \quad 22 \quad 6.497 \mathrm{E}+02 \quad 1.5 \%$

$27 \mathrm{CO} \quad 60 \quad 2.079 \mathrm{E}+02 \quad 2.3 \%$

$55 C S \quad 137 \quad 8.292 E+02 \quad 4.5 \%$

57LA $140 \quad 4.517 E+01 \quad 5.8 \%$

$635 U \quad 154 \quad 1.829 E+03 \quad 1.5 \%$

60. 8.MAY-1996 9:52:59 -50017 .

$\begin{array}{llll}11 \mathrm{NA} & 22 & \mathrm{~B} .695 \mathrm{E}+00 \quad 10.7 \%\end{array}$

$27 \mathrm{CO} \quad 60 \quad 3.971 \mathrm{E}+00 \quad 12.3 \%$

$55 \mathrm{CS} 137 \quad 4.939 E+01 \quad 12.1 \%$

57LA $140 \quad 4.341 E-01 \quad 68.4 \%$

63EU $154 \quad 2.448 E+01 \quad 10.7^{\circ}$

60. 8-MAY-1996 9:54:08 -48849 .

$11 \mathrm{NA} 22 \quad 1,272 \mathrm{E}+02 \quad 3.5 \%$

$27 \mathrm{CO} 60 \quad 3.765 \mathrm{E}+01 \quad 4.8 \%$

55CS $137 \quad 1.348 \mathrm{E}+02 \quad 9.9 \%$

57LA $140 \quad 8.783 E+00 \quad 10.1 \%$

G3EU $154 \quad 3.606 \mathrm{E}+02 \quad 3.4 \%$

60. 8.MAY-1996 $9: 55: 17 \quad-476 B 5$

$11 \mathrm{NA} 22 \quad 7.213 E+02 \quad 5.3 \%$

$27 C O \quad 60 \quad 2.460 E+02 \quad 3.0 \%$

47AGM $108 \quad 7.250 E+01 \quad 16.8 \%$

$55 \mathrm{CS} \quad 137 \quad 1.106 \mathrm{E}+03 \quad 3.9 \%$

57LA $140 \quad 5.175 E+01 \quad 4.5 \%$

63EU $154 \quad 2.031 E+03 \quad 5.3 \%$

8OHG $197 \quad 5.022 E+04 \quad 25.1 \%$

60. 8-MAY-1996 9:55:23 $\quad-48179$.

$11 \mathrm{NA} 22 \quad 4,239 \mathrm{E}+02 \quad 2.2 \%$

$27 \mathrm{CO} \quad 60 \quad 1.500 \mathrm{E}+02 \quad 2.5 \%$

55CS $137 \quad 6.426 \mathrm{E}+02 \quad 3.8 \%$

57LA $140 \quad 2.999 E+01 \quad 5.3 \%$

63EU $154 \quad 1.193 E+03 \quad 2.2 \%$

60. 8.MAY-1996 9:57:32 -49379.

$11 \mathrm{NA} 22 \quad 4.732 \mathrm{E}+02 \quad 1.6 \%$

$27 C 0 \quad 60 \quad 1.594 E+02 \quad 2.8 \%$

47AGM $108 \quad 7.875 E+01 \quad 15.0 \%$

$55 \mathrm{CS} 1376.029 \mathrm{E}+02 \quad 4.1 \%$

57LA $140 \quad 3.209 E+01 \quad 7.1 \%$

59PR $144 \quad 1.977 E+02 \quad 100.7 \%$

63EU $154 \quad 1.332 E+03 \quad 1.6 \%$

60. B-MAY-1996 9:58:38 -50535 .

$11 \mathrm{NA} \quad 22 \quad 1.160 \mathrm{E}+01 \quad 9.1 \%$

$27 C 0 \quad 60 \quad 3.843 E+00 \quad 12.4 \%$

55CS $137 \quad 4,114 E+01 \quad 14,4 \%$

63EU $154 \quad 3,266 \mathrm{E}+01 \quad 9,1 \%$

60. B-MAY-1996 $9: 59: 47 \quad-51730$.

11NA $22 \quad 8,099 E+01 \quad 3.2 \%$

$\begin{array}{llll}27 C 0 & 60 & 2.569 E+01 & 5.4 \%\end{array}$

$\begin{array}{llll}65 C S & 137 & 1.270 E+02 & 8.8 \%\end{array}$

57LA $140 \quad 1.246 E+00 \quad 56.2 \%$

G3EU $154 \quad 2.245 E+02 \quad 3.1 \%$

60. B-MAY-1996 10:00:56 -52939.

$11 \mathrm{NA} \quad 22 \quad 1.170 \mathrm{E}+02 \quad 3.2 \%$ 
WHC-SD-W151-CN-001, Rev. 0 Page 15 of 29

\begin{tabular}{|c|c|c|c|c|c|c|}
\hline & & & $27 \mathrm{CO}$ & 60 & $3.507 E+01$ & $4.6 \%$ \\
\hline & & & 358R & 84 & $2.240 E+00$ & $83.3 \%$ \\
\hline & & & $55 \mathrm{cs}$ & 137 & $1.707 E+02$ & $8.7 \%$ \\
\hline & & & 57LA & 140 & $6.465 E+00$ & $16.5 \%$ \\
\hline & & & 63EU & 154 & $3.296 E+02$ & $3.1 \%$ \\
\hline & & & 93NP & 239 & $9.469 E+05$ & $9.8 \%$ \\
\hline A2650810010 & 50896 10:01:03 & 60. & 8-MAY- 1996 to & 0:02:05 & $05 \quad-54000$. & \\
\hline & & & 11NA & 22 & $3.644 E+\infty 0$ & $16.0 \%$ \\
\hline & & & $27 \mathrm{CO}$ & 60 & $1.356 E+\infty$ & $21.0 \%$ \\
\hline & & & 63EU & 154 & $1.026 \mathrm{E}+0 t$ & $16.0 \%$ \\
\hline A2650810020 & 50896 10:02:10 & 60. & 8.MAY 199690 & 0:03:1 & $11 \quad-55294$. & \\
\hline & & & $27 \mathrm{CO}$ & 60 & $1.323 E+\infty$ & $23.3 \%$ \\
\hline & & & $80 \mathrm{HG}$ & 197 & $9.851 E+03$ & $61.5 \%$ \\
\hline A2650810030 & 50896 10:03:16 & 60. & 8-MAY-1996 10 & $0: 04: 17$ & $47-56437$ & \\
\hline & & & I1NA & 22 & $2.414 E+\infty 0$ & $36.6 \%$ \\
\hline & & & $55 \mathrm{cs}$ & 137 & $2.192 E+01$ & $17.1 \%$ \\
\hline & & & 63EU & 154 & $6.798 E+00$ & $36.6 \%$ \\
\hline A2650810040 & $50896 \quad 10: 04: 23$ & 60. & 8-MAY-1996 10 & $0: 05: 25$ & $23-57598$ & \\
\hline & & & $11 \mathrm{NA}$ & 22 & $3.075 E+00$ & $24.5 \%$ \\
\hline & & & $55 \mathrm{cs}$ & 137 & $2.641 E+01$ & $15.3 \%$ \\
\hline & & & 63EU & 154 & $8.657 E+\infty 0$ & $24.5 \%$ \\
\hline A2650810050 & $50896 \quad 10: 05: 31$ & 60. & 8.MAY-1996 10 & $0: 06: 36$ & 3357854. & \\
\hline & & & 11 NA & 22 & $3.605 E+00$ & $16.9 \%$ \\
\hline & & & $27 \mathrm{CO}$ & 60 & $1.610 E+\infty 0$ & $19.3 \%$ \\
\hline & & & 63EU & 154 & $1.015 E+01$ & $16.9 \%$ \\
\hline A2650810060 & $50896 \quad 10: 06: 39$ & 60. & 8-MAY-1996 10 & $0 ; 07: 39$ & $39-57854$. & \\
\hline & & & I1NA & 22 & $3.376 E+00$ & $16.6 \%$ \\
\hline & & & $27 \mathrm{co}$ & 60 & $1.417 E+00$ & $20.9 \%$ \\
\hline & & & 46PD & 109 & $2.201 E+04$ & $127.0 \%$ \\
\hline & & & $48 C D$ & 109 & $2.176 E+04$ & $127.0 \%$ \\
\hline & & & $55 \mathrm{cs}$ & 137 & $1.660 E+01$ & $20.8 \%$ \\
\hline & & & 63EU & 154 & $9.507 E+00$ & $16.6 \%$ \\
\hline A.2650810070 & $50896 \quad 10: 07: 49$ & 60. & 8.MAY-1996 10 & $0: 08: 51$ & $51-67854$ & \\
\hline & & & $11 \mathrm{NA}$ & 22 & $3,396 E+\infty 0$ & $16.6 \%$ \\
\hline & & & $\begin{array}{l}27 \mathrm{Co} \\
65 \mathrm{Cs}\end{array}$ & 60 & $2.470 E+00$ & $18.5 \%$ \\
\hline & & & 63EU & 154 & $9.561 E+00$ & $\begin{array}{l}17.5 \% \\
16.6 \%\end{array}$ \\
\hline A2650810100 & 50896 10:10:05 & 60. & 8-MAY-199610 & $0: 19: 0$ & $06 \quad-56708$ & \\
\hline & & & $27 \mathrm{CO}$ & 60 & $1.087 E+00$ & $33.4 \%$ \\
\hline & & & $39 Y$ & 912 & $2.252 \mathrm{E}+\mathrm{O} 2$ & $43.6 \%$ \\
\hline & & & $55 \mathrm{Cs}$ & 137 & $1.759 \mathrm{E}+01$ & $20.2 \%$ \\
\hline A2650810110 & $50896 \quad 10: 11: 14$ & 60. & 8-MAY-199610 & $0: 12: 1$ & $15 \quad-55548$ & \\
\hline & & & $11 \mathrm{NA}$ & 22 & $2.863 E+00$ & $17.5 \%$ \\
\hline & & & $27 \mathrm{CO}$ & 60 & 1. $393 E+00$ & $25.0 \%$ \\
\hline & & & $55 \mathrm{cs}$ & 137 & $3.288 E+01$ & $13.2 \%$ \\
\hline & & & 63EU & 154 & $8.062 E+00$ & $17.5 \%$ \\
\hline A2650810120 & $50896 \quad 10: 12 ; 21$ & 60. & 8.MAY-1996 10 & $0: 13: 2$ & $21 \quad-55147$. & \\
\hline & & & $11 \mathrm{NA}$ & 22 & $3.129 E+00$ & $20.8 \%$ \\
\hline & & & $27 \mathrm{CO}$ & 60 & $1.788 E+\infty$ & $25.3 \%$ \\
\hline & & & $55 \mathrm{Cs}$ & 137 & $3.035 E+01$ & $16,5 \%$ \\
\hline & & & G3EU & 154 & $8.811 E+00$ & $20.8 \%$ \\
\hline A2650810130 & $5089610: 13: 28$ & 60. & 8.MAY-199610 & $0: 14: 3 C$ & $30 \quad .56345$ & \\
\hline & & & $11 N A$ & 22 & $2.316 E+00$ & $25.6 \%$ \\
\hline & & & $27 \mathrm{CO}$ & 60 & $1.571 E+\infty 0$ & $23.0 \%$ \\
\hline & & & 40ZR & 97 & $1.988 \mathrm{E}+01$ & $63.8 \%$ \\
\hline & & & 55Cs & 137 & $2.494 \mathrm{E}+01$ & $15.9 \%$ \\
\hline & & & 63EU & 154 & $6.522 \mathrm{E}+00$ & $25.6 \%$ \\
\hline A2650810140 & $5089610: 14: 32$ & 60 & 8-MAY-199610 & $0: 15: 3$ & $33 \quad-57455$, & \\
\hline & & & 11NA & 22 & $2.007 E+00$ & $23.0 \%$ \\
\hline & & & $27 c 0$ & 60 & $1.596 \mathrm{E}+\infty 0$ & $22.9 \%$ \\
\hline & & & $55 \mathrm{cs}$ & 137 & $2.499 E+01$ & $15.9 \%$ \\
\hline & & & 63EU & 154 & $5.650 E+00$ & $23.0 \%$ \\
\hline A2650810160 & 50896 10:16:36 & 60. & 8-MAY-1996 10 & $0: 16: 0$ & -58547 & \\
\hline & & & $13 N A$ & 22 & $2.643 E+00$ & $18.7 \%$ \\
\hline & & & 63EU & 154 & $7.442 E+00$ & $18.7 \%$ \\
\hline
\end{tabular}


WHC-SD-W151-CN-001, Rev. 0

Page 16 of 29

A2650810170 50896 10:17:02

60. 8-MAY-1996 10:18:04 -60085.

$\begin{array}{lll}11 N A & 22 & 3.251 E+01 \quad 5.2 \%\end{array}$

20CA $47 \quad 9.832 \mathrm{E} \cdot 01 \quad 44.3 \%$

$27 C 0 \quad 60 \quad 1.169 E+01 \quad 8.4 \%$

55CS $137 \quad 4.711 E+01 \quad 13,0 \%$

E7LA $\quad 140 \quad 1.747 E+00 \quad 24.3 \%$

G3EU $154 \quad 9.154 \mathrm{E}+01 \quad 5.1 \%$ 


\section{Detector A3}

A3650808140 50896 08:14:32

A3650808150 50896 08:15:41

A $3650808160 \quad 5089608: 16: 50$

A $3650808170 \quad 5089608: 17: 57$

A3650808190 50896 08:19:06

A $3650808200 \quad 50896$ 08:20:13

A $3650808210 \quad 5089608: 21: 21$

A $3650808220 \quad 5089608: 22: 31$

A3650808230 50896 08:23:34

A3650808240 50896 08:24:40

A $3650808260 \quad 50896$ 08:26:49

А3650808300 50896 08:30:51

A $3650808310 \quad 50896$ 08:31:58

A3650808360 50896 08:36:33

A3650808370 50896 08:37:40

A3650808380 $5089608: 38: 48$

A.3650808410 50896 08:41:23

A3650808420 $5089608: 42: 30$

A3650808430 50896 08:43:36

A3650808440 50896 08:44:45

A $3650808450 \quad 50896$ 08:45:54

A3650808470 50896 08:47:00

A3650808480 50896 08:48:07

A3650808490 50896 08:49:15

A365080B500 50896 08:50:22

A3650808510 50896 08:51:30

A3650808520 50896 08:52:38

A3650808530 50896 08:53:47
60. B-MAY-1996 8:15:34 -10940. $55 \mathrm{Cs} 137 \quad 1.753 E-04 \quad 5.7 \%$ 60. 8.MAY-1996 8:16:43 11187.

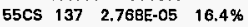

60. 8.MAY-1996 8:17:62 -11187 . 55 CS $137 \quad 2.258 E-05 \quad 18.5 \%$

60. B-MAY-1996 8:18:58 +11187. 55CS $137 \quad 1.694 E-05 \quad 19.7 \%$

60. 8-MAY-1996 8:20:07 -11187. $55 \mathrm{Cs} \quad 137 \quad 2.506 \mathrm{E}-05 \quad 16.3 \%$

60. 8-MAY-1996 8:21:13 -11187. 55CS $137 \quad 2.543 E-05 \quad 16.0 \%$

60, 8-MAY-1996 8:22:22 + 11187 . 55CS $137 \quad 2.268 E-05 \quad 17.4 \%$

60. 8-MAY-1996 8:23:31 - 11187 . 55CS $137 \quad 3.370 E-05 \quad 14.7 \%$

60. 8-MAY-1996 8:24:34 - 11187 . 55 Cs $137 \quad 2.416 \mathrm{E}-05 \quad 16.3 \%$

60. 8.MAY-1996 8:25:42 -11187 $55 \mathrm{Cs} \quad 137 \quad 2.282 \mathrm{E}-05 \quad 20.4 \%$

60. 8-MAY-1996 6:27:49 -11187. 55CS $137 \quad 2.242 \mathrm{E}-05 \quad 17.6 \%$

60. 8.MAY-1996 8:31:52 -11930. 55CS $137 \quad 3.733 E-05 \quad 13.2 \%$

60. 8-MAY-1996 8:32:58 -13011 55CS $137 \quad 5.627 E-05 \quad 10.4 \%$

60. 8.MAY-1996 8:37:34 -17509. $55 \mathrm{CS} \quad 137 \quad 2.843 \mathrm{E}-05 \quad 15.4 \%$

60, 8-MAY-1996 8:38:40 -17784. $55 \mathrm{CS} 137 \quad 6.011 \mathrm{E}-05 \quad 10.6 \%$

60. 8.MAY-1996 8:39:49 -17784. $55 \mathrm{CS} \quad 137 \quad 7.388 \mathrm{E}-05 \quad 9,3 \%$

60. 8.MAY-1996 B:42:25 18926 55CS $137 \quad 3.674 \mathrm{E}-05 \quad 13.3 \%$

60. 8-MAY-1996 8:43:31 -19998. $55 \mathrm{CS} \quad 137 \quad 4.592 \mathrm{E}-05 \quad 11.9 \%$

60. 8-MAY-1996 8:44:37 -21082. 55CS $137 \quad 5.044 \mathrm{E}-05 \quad 11.4 \%$

60. 8-MAY-1996 8:45:46 -22203 56CS $137 \quad 7.372 E-05 \quad 9.0 \%$

60. 8-MAY-1996 8:46:55 $\quad-23333$ $55 \mathrm{CS} \quad 137 \quad 1.542 \mathrm{E}-04 \quad 6.1 \%$

60. B-MAY-1996 B:48:01 -24405. 55CS $137 \quad 8.937 \mathrm{E}-04 \quad 2.5 \%$

60. B-MAY-1996 8:49:07 -25489. 55CS $134 \quad 4.756 E+00 \quad 26.0 \%$ $55 \mathrm{CS} 137 \quad 1.525 \mathrm{E}-03 \quad 1.9 \%$

60. 8-MAY-1996 B:50:17 -26627 55CS $134 \quad 6.224 \mathrm{E}+00 \quad 22.7 \%$ 55CS $137 \quad 2.226 E-03 \quad 2.1 \%$

60. B-MAY-1996 8:51:23 $\quad-27700$ 55CS $134 \quad 5.507 E+00 \quad 30.9 \%$ 55CS $137 \quad 1.875 E .03 \quad 2.1 \%$

60. B-MAY-1996 8:52:30 -28795. 55CS $137 \quad 1.129 \mathrm{E}-03 \quad 2,2 \%$

60. 6-MAY-1996 8:53:39 -29924 . 11NA $22 \quad 3.871 \mathrm{E}+00 \quad 30.4 \%$ 52TEM $127 \quad 7.197 E .03 \quad 33.7 \%$ 55CS $134 \quad 2.751 E+00 \quad 38.9 \%$ 55CS $137 \quad 1.318 \mathrm{E}-03 \quad 3.4 \%$ G3EU $154 \quad 1.090 E+01 \quad 30.4 \%$ $92 \mathrm{U} \quad 232 \quad 1.835 \mathrm{E}-02 \quad 33.7 \%$

60. B-MAY-1996 8:54:48 -31046 . IINA $22 \quad 6.167 \mathrm{E}+01 \quad 6.8 \%$ 
WHC-SD-W151-CN-001, Rev. 0

Page 18 of 29

A3650808540 50896 08:54:53

A3650808550 50896 08:55:59

A3650808570 50896 08:57:05

A3650808580 50896 08:58:11

A3650808590 50896 08:59:19

A3650809000 50896 09:00:26

A3650809010 50896 09:01:32

A3650809020 50896 09:02:41

A3650809030 50896 09:03:49
$27 \mathrm{CO} \quad 60 \quad 1,645 E+01 \quad 11.0 \%$

$55 \mathrm{CS} \quad 134 \quad 6.438 \mathrm{E}+00 \quad 88.6 \%$ 55CS $137 \quad 2.329 E-03 \quad 2.1 \%$

57LA $140 \quad 3.684 E+\infty 0 \quad 29.1 \%$

63EU $154 \quad 1.680 E+02 \quad 6.4 \%$

60. 8-MAY-1996 8:55:54 -32123.

1INA 22 5.B27E + 02, 2.3\%

$27 \mathrm{CO} \quad 60 \quad 2.563 E+02 \quad 3.6 \%$

46PD $109 \quad 3.895 E-04 \quad 139.5 \%$

48CD $109 \quad 3.851 E-04 \quad 139.5 \%$

55CS 137 4.683E-03 $1.2 \%$

57LA $140 \quad 4.253 E+01 \quad 11.0 \%$

G3EU $154 \quad 1.645 E+03 \quad 2.3 \%$

60. 8-MAY-1996 8:57:00 -31881.

11NA $22 \quad 1.102 E+03 \quad 1.8 \%$

$27 \mathrm{CO} \quad 60 \quad 4.590 \mathrm{E}+02 \quad 2.7 \%$

4OZR $95 \quad 1.367 E+03 \quad 25.0 \%$

$41 \mathrm{NB} \quad 95 \quad 5.332 \mathrm{E}+02 \quad 45.0 \%$

$\begin{array}{llll}55 \mathrm{CS} & 137 & 6.929 \mathrm{E}-03 & 1.4 \%\end{array}$

57LA $140 \quad 7.202 E+01 \quad 7.2 \%$

63EU $154 \quad 3.113 E+03 \quad 1.8 \%$

60. 8-MAY-1996 8:58:06 -30832,

$11 N A \quad 22 \quad 3,203 E+02 \quad 3.4 \%$

$27 \mathrm{CO} \quad 60 \quad 1.069 E+02 \quad 4.4 \%$

55CS $137 \quad 3.913 E-03 \quad 1.3 \%$

57LA $140 \quad 2.012 E+01 \quad 15.1 \%$

G3EU $154 \quad 9.036 E+02 \quad 3,3 \%$

60. 8-MAY-1996 8:59:12 -30091.

$11 \mathrm{NA} \quad 22 \quad 2.80 \mathrm{BE}+01 \quad 19.4 \%$

$27 \mathrm{CO} \quad 60 \quad 9.000 E+00 \quad 14.7 \%$

52TEM $127 \quad 1.086 E-02 \quad 30.5 \%$

$55 \mathrm{CS} 137 \quad 1.814 \mathrm{E}-03 \quad 3.1 \%$

63EU $154 \quad 6.824 E+01 \quad 9.6 \%$ $92 \mathrm{U} \quad 232 \quad 2.770 \mathrm{E}-02 \quad 30.5 \%$

60. E-MAY-1996 9:00:2t -29472

$11 \mathrm{NA} 22 \quad 3.645 \mathrm{E}+00 \quad 33.9 \%$

$\begin{array}{lll}55 C S \quad 134 & 5.047 E+00 \quad 29.8 \%\end{array}$

55CS $137 \quad 1.403 E-03 \quad 2.5 \%$

63EU $154 \quad 1.026 \mathrm{E}+01 \quad 33,9 \%$

60. 8.MAY-1996 9:01:27 $\quad-30542$.

$\begin{array}{llll}11 \mathrm{NA} & 22 & 1.570 \mathrm{E}+01 \quad 13.6 \%\end{array}$

$27 \mathrm{CO} \quad 60 \quad 4,312 \mathrm{E}+00 \quad 22.6 \%$

52TEM $127 \quad 9.205 E-03 \quad 32.8 \%$

$55 \mathrm{Cs} 134 \quad 3.615 \mathrm{E}+00 \quad 62.2 \%$

55CS 137 1.529E-03 $1.9 \%$

G3EU $154 \quad 4.377 E+01 \quad 11.8 \%$

$92 \mathrm{U} \quad 232 \quad 2.347 \mathrm{E}-02 \quad 32.8 \%$

60. 8-MAY-1996 9:02:33 -31620 .

$\begin{array}{llll}11 \mathrm{NA} & 22 & 2.107 \mathrm{E}+02 & 4.9 \%\end{array}$

$\begin{array}{llll}27 \mathrm{CO} & 60 & 6.737 E+01 \quad 5.2 \%\end{array}$

52TEM $127 \quad 1.818 E-02 \quad 44.7 \%$

$531 \quad 135 \quad 2.474 E+01 \quad 166.9 \%$

55CS $137 \quad 3.696 \mathrm{E}-03 \quad 1.6 \%$

57LA $140 \quad 1.694 E+01 \quad 18.9 \%$

63EU $154 \quad 6.786 E+02 \quad 4.8 \%$

$92 \mathrm{U} \quad 232 \quad 4.637 \mathrm{E}-02 \quad 44.7 \%$

60. 8-MAY-1996 $9: 03: 42 \quad-32729$.

$\begin{array}{lll}11 \mathrm{NA} & 22 & 1.005 \mathrm{E}+03 \quad 2.8 \%\end{array}$

$27 \mathrm{CO} \quad 60 \quad 4.259 \mathrm{E}+02 \quad 3.2 \%$

55CS 137 5.815E-03 1.2\%

57LA $140 \quad 6.538 E+01 \quad 7.9 \%$

63EU $154 \quad 2.846 E+03 \quad 2.7 \%$

60. B-MAY-1996 9:04:51 -33860 .

54XEM $135 \quad 1.387 E-04 \quad 41.5 \%$

55CS $137 \quad 7.481 E-02 \quad 2.3 \%$

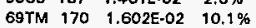




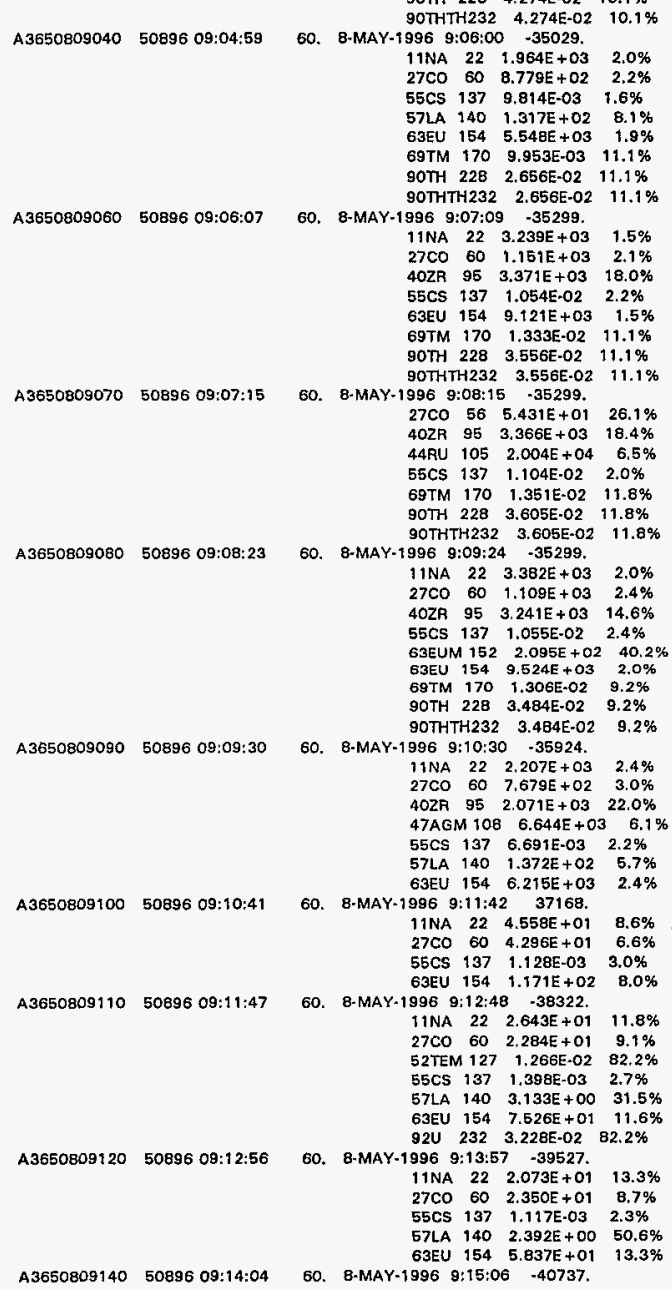
90THTH $232 \quad 4.274 E-02 \quad 10.1 \%$ 60. 8-MAY-1996 9:06:00 -35029.

$\begin{array}{llll}11 \mathrm{NA} \quad 22 & 9.964 \mathrm{E}+03 \quad 2.0 \%\end{array}$ $\begin{array}{llll}27 C O & 60 & 8.779 E+02 & 2.2 \%\end{array}$ 55CS $137 \quad 9.814 E-03 \quad 1.6 \%$ 57LA $140 \quad 1.317 E+02 \quad 8.1 \%$ G3EU $154 \quad 5.548 E+03 \quad 1.8 \%$ G9TM $170 \quad 9.953 \mathrm{E}-03 \quad 11.1 \%$ 90TH $228 \quad 2.656 \mathrm{E}-02 \quad 11.1 \%$ 9OTHTH $232 \quad 2.656 \mathrm{E}-02 \quad 11.1 \%$ 60. 8-MAY-1996 9:07:09 -35299.

11NA $22 \quad 3.239 \mathrm{E}+03 \quad 1.5 \%$ $\begin{array}{llll}27 C 0 & 60 & 1.151 E+03 \quad 2.1 \%\end{array}$ 40ZR $95 \quad 3.371 \mathrm{E}+03 \quad 18.0 \%$ $\begin{array}{llll}55 C S & 137 & 1.054 E-02 & 2.2 \%\end{array}$ G3EU $154 \quad 9.121 E+03 \quad 1.5 \%$ 69TM $170 \quad 1.333 E-02 \quad 11.1 \%$ 90TH $228 \quad 3.556 E-02 \quad 11.1 \%$ 9OTHTH $232 \quad 3.556 E-02 \quad 11.1 \%$

60. 8-MAY-1996 9:08:15 -35299. $27 \mathrm{CO} \quad 56 \quad 5.431 E+01 \quad 26.1 \%$ 4OZR $95 \quad 3.366 E+03 \quad 18.4 \%$ 44RU $105 \quad 2.004 E+04 \quad 6.5 \%$ 55CS $137 \quad 1.104 \mathrm{E} .02 \quad 2.0 \%$ 69TM $170 \quad 1.351 E-02 \quad 11.8 \%$ 90TH $228 \quad 3.605 E-02 \quad 11.8 \%$ 90THTH $232 \quad 3.605 E .02 \quad 11.8 \%$

60. 8-MAY-1996 9:09:24 -35299.

11NA $22 \quad 3.382 E+03 \quad 2.0 \%$ $\begin{array}{llll}27 C 0 & 60 & 1.109 E+03 \quad 2.4 \%\end{array}$ 40ZA $95 \quad 3.241 \mathrm{E}+03 \quad 14.6 \%$ $\begin{array}{llll}55 C S & 137 & 1.055 E-02 & 2.4 \%\end{array}$ G3EUM $152 \quad 2.095 E+02 \quad 40.2 \%$ G3EU $154 \quad 9.524 E+03 \quad 2.0 \%$ 69TM $170 \quad 1.306 \mathrm{E}-02 \quad 9.2 \%$ 9OTH $228 \quad 3.484 E-02 \quad 9.2 \%$ 90THTH232 3.484E-02 $\quad 9.2 \%$

60. 8-MAY-1996 9:10:30 -35924.

I1NA $22 \quad 2,207 E+03 \quad 2,4 \%$ $27 C 0 \quad 60 \quad 7,679 E+02 \quad 3.0 \%$ 402月 $95 \quad 2.071 E+03 \quad 22.0 \%$ 47AGM $108 \quad 6.644 E+03 \quad 6.1 \%$ 55CS $137 \quad 6.691 \mathrm{E}-03 \quad 2.2 \%$ 57LA $140 \quad 1.372 E+02 \quad 5.7 \%$ G3EU $154 \quad 6.215 E+03 \quad 2.4 \%$

60. B-MAY-1996 9:11:42 37168. $11 \mathrm{NA} 22 \quad 4.558 \mathrm{E}+01 \quad 8.6 \%$ $27 \mathrm{CO} \quad 60 \quad 4.296 \mathrm{E}+01 \quad 6.6 \%$ $55 \mathrm{CS} \quad 137 \quad 1.128 \mathrm{E}-03 \quad 3.0 \%$ GSEU $154 \quad 1.171 E+02 \quad 8.0 \%$ 60. 8-MAY-1996 9:12:48 $\quad$-38322. I1NA $22 \quad 2.643 \mathrm{E}+01 \quad 11.8 \%$ $27 C 0 \quad 60 \quad 2,284 E+01 \quad 9.1 \%$ $\begin{array}{llll}\text { 52TEM } 127 & 1,266 \mathrm{E}-02 & 82.2 \%\end{array}$ $\begin{array}{llll}55 C S & 137 & 1.398 E-03 & 2.7 \%\end{array}$ 57LA $140 \quad 3.133 E+00 \quad 31.5 \%$ G3EU $154 \quad 7.526 E+01 \quad 11.6 \%$ $920 \quad 232 \quad 3.228 \mathrm{E}-02 \quad 82.2 \%$

60. 8-MAY-1996 9:13:57 -39527. 11NA $22 \quad 2.073 \mathrm{E}+01 \quad 13.3 \%$ $\begin{array}{llll}27 C O & 60 & 2.350 E+01 & 8.7 \%\end{array}$ 55CS $137 \quad 1.117$ E-03 $2.3 \%$ E7LA $140 \quad 2.392 E+00 \quad 50,6 \%$ 63EU $154 \quad 5.837 E+01 \quad 13.3 \%$ 60. 8-MAY-1996 9:15:06 -40737.

90TH $228 \quad 4,274 E-02 \quad 10.1 \%$ 
WHC-SD-W151-CN-001, Rev. 0

Page 20 of 29

A3650809150 50896 09:15:11

A3650809160 50896 09:16:20

A3650809170 50896 09:17:26

A $3650809180 \quad 50896$ 09:18:36

A $3650809190 \quad 50896$ 09:19:44

A3650809210 50896 09:21:59

A3850809230 50896 09:23:05

A3650809240 $50896 \quad 09: 24: 11$

A3650809250 50896 09:25:18

A3650809260 50896 09:26:27
11NA $22 \quad 2.351 \mathrm{E}+01 \quad 12.0 \%$ $\begin{array}{llll}27 C O & 60 & 2.091 E+01 & 9.7 \%\end{array}$ $\begin{array}{llll}55 C S & 137 & 1.456 E-03 & 2.0 \%\end{array}$ 57LA $140 \quad 2.149 E+00 \quad 53.3 \%$ 63EU $154 \quad 6.662 E+01 \quad 10.4 \%$ 60. 8-MAY-1996 9:16:12 -41889.

1INA $22 \quad 2.439 E+01 \quad 13.8 \%$ 20CA $47 \quad 3.441 E+\infty \quad 97.5 \%$ $\begin{array}{llll}27 \mathrm{CO} & 60 & 1.740 \mathrm{E}+01 \quad 10.7 \%\end{array}$

$\begin{array}{llll}55 C S & 137 & 1.252 E-03 & 2.2 \%\end{array}$

57LA $140 \quad 3.719 E+00 \quad 36.3 \%$ 63EU $154 \quad 6.696 E+01 \quad 11.5 \%$

60. 8-MAY-1996 9:17:21 +43095 .

11NA $22 \quad 2.372 E+01 \quad 12.0 \%$ $27 \mathrm{CO} \quad 60 \quad 2.433 E+01 \quad 9.5 \%$ $\begin{array}{llll}\text { 55CS } 137 & 1.223 \mathrm{E} .03 & 2.2 \%\end{array}$ GSEU $154 \quad 6.679 E+01 \quad 12.0 \%$

60. 8-MAY-1996 9:18:27 -44246. 11NA $22 \quad 2.184 \mathrm{E}+01 \quad 15.4 \%$

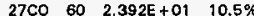
$\begin{array}{llll}55 C S \quad 137 & 1.022 E-03 \quad 2.4 \%\end{array}$ $\begin{array}{llll}\text { S7LA } & 140 & 2.761 E+00 \quad 34.3 \%\end{array}$ 63EU $154 \quad 6.272 E+01 \quad 14.2 \%$

50. 8.MAY-1996 9:19:36 -44495,

11NA $22 \quad 2.404 \mathrm{E}+01 \quad 16.5 \%$ $\begin{array}{llll}27 \mathrm{CO} & 60 & 1.967 \mathrm{E}+01 \quad 10.0 \%\end{array}$ $\begin{array}{llll}55 C S \quad 137 & 1.171 E-03 \quad 2.3 \%\end{array}$ 63EU $154 \quad 5.985 E+01 \quad 14.1 \%$

60. 8-MAY.1996 $\quad 9 ; 20: 45 \quad-43328$

$\begin{array}{llll}11 \mathrm{NA} & 22 & 1.905 \mathrm{E}+01 & 13.6 \%\end{array}$ $27 \mathrm{CO} \quad 60 \quad 1.970 \mathrm{E}+01 \quad 11.6 \%$ $39 Y \quad 95 \quad 2.620 \mathrm{E}+01 \quad 51.6 \%$ 55CS $137 \quad 9.363 \mathrm{E}-04 \quad 2.5 \%$ 63EU $154 \quad 5.363 E+01 \quad 13.6 \%$

60. 8-MAY-1996 9:21:54 -42154

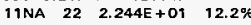
$27 C 0 \quad 60 \quad 2.138 E+01 \quad 9,8 \%$ 52TEM $127 \quad 1.155 \mathrm{E}-02 \quad 37.9 \%$ 55CS $137 \quad 1.297 \mathrm{E}-03 \quad 3.4 \%$ 63EU $154 \quad 5.895 E+01 \quad 11.6 \%$ $92 \mathrm{U} \quad 232 \quad 2.944 \mathrm{E}-02 \quad 37.9 \%$

60. 8-MAY-1996 9:23:00 -42506 . $11 \mathrm{NA} 22 \quad 2.933 \mathrm{E}+01 \quad 12.6 \%$ $\begin{array}{lllll}27 C 0 & 60 & 2.315 E+01 & 9.4 \%\end{array}$ $\begin{array}{lll}\text { 52TEM } 127 & 1.153 E-02 & 33.2 \%\end{array}$ $\begin{array}{llll}55 \mathrm{CS} & 137 \quad 1.257 \mathrm{E}-03 \quad 2.2 \%\end{array}$ 63EU $154 \quad 7.061 \mathrm{E}+01 \quad 11.1 \%$ $92 \mathrm{U} \quad 232 \quad 2.941 E-02 \quad 33.2 \%$

60. B-MAY-1996 9:24:06 -43663 $\begin{array}{lll}11 \mathrm{NA} & 22 & 2.498 \mathrm{E}+01 \quad 14.5 \%\end{array}$ $\begin{array}{llll}27 C 0 & 60 & 2.033 E+01 & 9.9 \%\end{array}$ $\begin{array}{llll}55 C S & 137 & 1.236 E-03 & 2.2 \%\end{array}$ 63EU $154 \quad 7.033 E+01 \quad 14.5 \%$

60. 8-MAY-1996 9:25:12 -44812

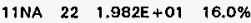
$27 C 0 \quad 60 \quad 2.153 E+01 \quad 9.8 \%$ $\begin{array}{llll}55 C 5 & 137 & 9.730 E-04 \quad 2.8 \%\end{array}$ G3EU $154 \quad 5.580 E+01 \quad 16.0 \%$

60. 8-MAY-1996 9:26:18 -45973.

11NA $22 \quad 3.101 E+02 \quad 3.9 \%$ $27 C 0 \quad 60 \quad 1.193 E+02 \quad 4.6 \%$ $\begin{array}{llll}55 C S & 137 & 2.236 E-03 & 2.2 \%\end{array}$ 57LA $140 \quad 2,279 E+01 \quad 12.5 \%$ 59PR $144 \quad 7.323 E+02 \quad 65.7 \%$ G3EU $154 \quad 8.785 E+02 \quad 3.8 \%$

60. 8-MAY-1996 9:27:27 $\quad-45583$. 
WHC-SD-W151-CN-001, Rev. 0

Page 21 of 29

A3650809270 50896 09:27:38

A3650809280 50896 09:28:44

A3650809290 50896 09:29:51

A3650809300 50896 09:30:58

A $3650809320 \quad 50896$ 09:32:06

A3650809330 50896 09:33:15

A3650809340 50896 09:34:23

A3650809350 50896 09:35:33

A3650809360 50896 09:36:41
11NA $22 \quad 1.448 \mathrm{E}+03 \quad 2.2 \%$

$27 \mathrm{CO} \quad 60 \quad 5.562 E+02 \quad 3.3 \%$

4OZR $95 \quad 1.329 E+03 \quad 23.4 \%$

47AGM $108 \quad 4.172 \mathrm{E}+03 \quad 10.6 \%$

55CS $137 \quad 7.194 \mathrm{E}-03 \quad 2.1 \%$

57LA $140 \quad 9.826 E+01 \quad 7.4 \%$

63EU $154,4.096 \mathrm{E}+03 \quad 2.2 \%$

BOHG $997 \quad 4.381 \mathrm{E}-04 \quad 30.9 \%$

60. 8-MAY-1996 9:28:39 -44357.

$\begin{array}{lll}11 \mathrm{NA} & 22 & 3.030 \mathrm{E}+01 \quad 10.7 \%\end{array}$

$27 \mathrm{CO} 60 \quad 2.726 E+01 \quad 11.9 \%$

55CS $137 \quad 1: 104 \mathrm{E}-03 \quad 3.2 \%$

63EU $154 \quad 8,525 E+01 \quad 10,3 \%$

60. 8.MAY-1996 9:29:45 -43240.

IINA $22 \quad 2.200 E+01 \quad 13.9 \%$

$27 \mathrm{CO} \quad 60 \quad 2.535 \mathrm{E}+01 \quad 9.5 \%$

55CS 137 1.113E-03 $2.4 \%$

63EU $154 \quad 5.595 E+01 \quad 12.8 \%$

60. 8-MAY-1996 $9: 30: 52 \quad-42277$.

11 NA $22 \quad 2.408 E+01 \quad 11.8 \%$

$27 \mathrm{CO} \quad 60 \quad 2.193 E+01 \quad 10.0 \%$

55CS $137 \quad 1.297 \mathrm{E}-03 \quad 2.2 \%$

63EU $154 \quad 6.781 E+01 \quad 11.8 \%$

60. 8.MAY-1996 $9: 31: 58 \quad-42277$.

$11 \mathrm{NA} 22 \quad 2.155 \mathrm{E}+01 \quad 13.8 \%$

$\begin{array}{llll}27 C O & 60 & 1.933 E+01 & 10.0 \%\end{array}$

$55 \mathrm{CS} \quad 137 \quad 1.209 \mathrm{E}+03 \quad 2.2 \%$

G3EU $154 \quad 5.507 E+01 \quad 12.7 \%$

60. 8-MAY+1996 9:33:07 $\quad .43274$.

IINA $22 \quad 2.224 E+01 \quad 14.1 \%$

$23 V \quad 52 \quad 2.465 E+00 \quad 47.9 \%$

$27 \mathrm{CO} \quad 60 \quad 2.227 \mathrm{E}+01 \quad 9.5 \%$

52TEM $127 \quad 7.530 E \cdot 03 \quad 137.4 \%$

$\begin{array}{llll}55 \mathrm{CS} & 137 & 1.268 \mathrm{E}-03 & 2.2 \%\end{array}$

63EUM 152 2.852E+01 $56.7 \%$

G3EU $154 \quad 6.320 E+01 \quad 13.2 \%$

92U $232 \quad 1.920 E-02 \quad 137.4 \%$

60. B-MAY-1996 $9: 34: 16 \quad-44478$.

$11 \mathrm{NA} \quad 22 \quad 2.136 \mathrm{E}+01 \quad 13.9 \%$

$2700 \quad 60 \quad 2.003 E+07 \quad 9.7 \%$

$\begin{array}{llll}55 \mathrm{CS} & 137 & 1.053 E .03 \quad 2.4 \%\end{array}$

G3EU $154 \quad 5.870 E+01 \quad 13.1 \%$

60. 8-MAY-1996 9:35:25 -45686.

$11 \mathrm{NA} 22 \quad 4,264 \mathrm{E}+01 \quad 9.3 \%$

$27 \mathrm{CO} \quad 60 \quad 3.016 \mathrm{E}+01 \quad 8.0 \%$

$29 \mathrm{CU} 66 \quad 4.264 \mathrm{E}+01 \quad 76.9 \%$

52TEM 131 2.177E + 01 58.5\%

55CS $137 \quad 1.106 \mathrm{E}-03 \quad 3.1 \%$

63EU $154 \quad 1.133 E+02 \quad 8.9 \%$

60. 8-MAY-1996 9:36:34 -46596 .

$\begin{array}{llll}11 N A & 22 & 1.543 E & +03 \quad 2.0 \%\end{array}$

$27 \mathrm{CO} \quad 60 \quad 5.389 E+02 \quad 3.6 \%$

4OZR $95 \quad 1.430 E+03 \quad 25.5 \%$

47AGM $108 \quad 4.255 E+03 \quad 8.4 \%$

$531 \quad 135 \quad 3.443 E+01 \quad 51.3 \%$

$55 \mathrm{CS} \quad 137 \quad 6.245 E=03 \quad 2.5 \%$

57LA $140 \quad 1.051 E+02 \quad 6.0 \%$

G3EU $154 \quad 4.343 E+03 \quad 2.0 \%$

60. 8-MAY-1996 $9: 37: 43 \quad-46596$,

$\begin{array}{llll}\text { I NA } 22 & 1.215 E+03 & 2.2 \%\end{array}$

$27 \mathrm{CO} \quad 60 \quad 4.389 \mathrm{E}+02 \quad 4.0 \%$

29CU $66 \quad 3.775 E+02 \quad 30.4 \%$

35BR $82 \quad 3.390 E+02 \quad 47.6 \%$

41 NBM $92 \quad 2.005 E+01 \quad 45.4 \%$

39Y $92 \quad 1.430 E+02 \quad 45.4 \%$

$\begin{array}{llll}40 Z R & 95 & 9.454 E+02 & 28.8 \%\end{array}$ 
WHC-SD-W151-CN-001, Rev. 0

Page 22 of 29

A3650809370 $50896 \quad 09: 37: 49$

A3650809380 50896 09:38:57

A 3650809400 50896 09:40:07

A $3650809410 \quad 50896$ 09:41:12

A $3650809420 \quad 5089609: 42: 24$

A3650809430 $5089609: 43: 33$

A3650809440 $5089609: 44: 42$

A3650809450 50896 09:45:51

A3650809460 50896 09:46:58
47AGM $108 \quad 2.699$ + $+03 \quad 10.4 \%$

55CS $137 \quad 4.168 E-03 \quad 1.9 \%$

57LA $140 \quad 7.951 E+01 \quad 7.1 \%$

63EU $154 \quad 3.436 E+03 \quad 2.1 \%$

60. 8-MAY-1996 9:38:51 -46596.

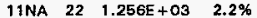

$27 C 0 \quad 60 \quad 4.496 E+02 \quad 3.6 \%$

47AGM IOB $3.312 E+03 \quad 8.7 \%$

46PD $109 \quad 2.966 E-03 \quad 28.9 \%$

55CS $137 \quad 4.187 E-03 \quad 1.4 \%$

57LA $140 \quad 8.652 E+01 \quad 8.0 \%$

G3EU $154 \quad 3.550 E+03 \quad 2.2 \%$

90TH $228 \quad 3.051 E-02 \quad 10.7 \%$

90THTH232 3.051E-02 $10.7 \%$

60. 8-MAY-1996 9:39:58 -47080

$11 \mathrm{NA} 22 \quad 1.211 \mathrm{E}+03 \quad 2.6 \%$

$27 \mathrm{CO} \quad 60 \quad 4.511 \mathrm{E}+02 \quad 2.8 \%$

47AGM $108 \quad 3.822 E+03 \quad 8.4 \%$

55CS $137 \quad 4.179 E-03 \quad 1.8 \%$

57LA $140 \quad 8.975 E+01 \quad 6.6 \%$

G3EU $154 \quad 3.409 E+03 \quad 2.6 \%$

60. 8-MAY-1996 9:41:07 -46101.

44RU $105 \quad 4.448 E+04 \quad 6.8 \%$

45RH $106 \quad 2.088 E-03 \quad 45.1 \%$

54XEM $135 \quad 3.153 \mathrm{E}-04 \quad 36.1 \%$

$\begin{array}{lll}55 \mathrm{CS} \quad 137 \quad 2.588 \mathrm{E}-02 & 3.2 \%\end{array}$

B3B! $212 \quad 5.209 \mathrm{E}+03 \quad 287.7 \%$

9OTHTH232 7.970E-02 $10.5 \%$

90 THBI232 $5.209 E+03 \quad 287.7 \%$

60. 8-MAY- $1996 \quad$ 9:42:13 $\quad-47139$.

$55 \mathrm{CS} \quad 137 \quad 2.181 \mathrm{E}-02 \quad 3.4 \%$

69TM $170 \quad 2.522 \mathrm{E}-02 \quad 12.8 \%$

90TH $228 \quad 6.730 \mathrm{E}-02 \quad 12.8 \%$

90THTH $232 \quad 6.730 E-02 \quad 12.8 \%$

60. B.MAY-1996 $9: 43: 25 \quad-45927$.

SF $20-2.832 \mathrm{E}+00 \quad 195.7 \%$

IINA $22 \quad 1.451 \mathrm{E}+03 \quad 2.0 \%$

$27 \mathrm{CO} \quad 60 \quad 5.134 \mathrm{E}+02 \quad 2.9 \%$

$40 Z R \quad 95 \quad 1.031 E+03 \quad 27.9 \%$

47AGM $108 \quad 3.752 E+03 \quad 8.7 \%$

$55 \mathrm{CS} \quad 137 \quad 5.578 \mathrm{E}-03 \quad 1.6 \%$

57LA $140 \quad 9.604 \mathrm{E}+01 \quad 6.2 \%$

63EU $154 \quad 4.088 E+03 \quad 2.0 \%$

60. 8-MAY-1996 9:44:34 .44750,

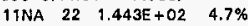

$\begin{array}{llll}27 \mathrm{CO} & 60 & 7.672 \mathrm{E}+01 \quad 6.7 \%\end{array}$

55CS $137 \quad 1.635 \mathrm{E}-03 \quad 2.3 \%$

57LA $140 \quad 1.123 E+01 \quad 17.6 \%$

63EU $154 \quad 4.084 E+02 \quad 4.6 \%$

60. 8-MAY-1996 $9: 45: 43 \quad-44377$.

11 NA $22 \quad 2.255 E+01 \quad 12.9 \%$

$27 \mathrm{CO} \quad 60 \quad 1.739 \mathrm{E}+01 \quad 11.0 \%$

52TEM 127 1.115E-02 45.7\%

55CS $137 \quad 9.381 E-04 \quad 2.5 \%$

63EU $154 \quad 6.34 B E+01 \quad 12.9 \%$

$92 \mathrm{U} \quad 232 \quad 2.645 \mathrm{E}-02 \quad 45.7 \%$

60. 8-MAY-1996 $9: 46: 53 \quad-45603$,

$11 \mathrm{NA} \quad 22 \quad 3.37 \mathrm{BE}+01 \quad 10.1 \%$

$2700 \quad 60 \quad 2.520 E+01 \quad 10.0 \%$

$55 \mathrm{CS} \quad 137 \quad 1.086 \mathrm{E}-03 \quad 2.4 \%$

63EU $154 \quad 9.069 E+01 \quad 9.3 \%$

60. 8-MAY-19:96 $9: 47: 59 \quad-46723$.

$\begin{array}{lll}11 \mathrm{NA} & 22 & 1.472 \mathrm{E}+03 \quad 3.0 \%\end{array}$

27CO $60 \quad 5.573 E+02 \quad 3.4 \%$

4OZR $95 \quad 1.303 E+03 \quad 27.2 \%$

47AGM $108 \quad 3.680 E+03 \quad 8.2 \%$ 
WHC-SD-W151-CN-001, Rev. 0

Page 23 of 29

A3550809480 50896 09:48:07

A3650809490 50896 09:49:13

A3650809510 50896 09:51:58

A3650809530 50896 09:53:07

A3650809540 50896 09:54:16

A3650809550 50896 09:55:22
60. B-MAY-1996 9:49:08 47018

55Cs $137 \quad 6.896 E-03 \quad 2.3 \%$

57LA $140 \quad 9.088 E+01 \quad 6.5 \%$

63EU $154 \quad 4.979 E+03 \quad 2.8 \%$

8OHO 197 1.593E-04 $94.8 \%$

90 THTH 232 2.635E-02 $13.3 \%$

$11 N A \quad 22 \quad 1.214 E+03 \quad 1.7 \%$

$27 C 0 \quad 60 \quad 4.440 E+02 \quad 4.0 \%$

4OZR $95 \quad 1.157 E+03 \quad 24.0 \%$

47AGM $108 \quad 3.213 E+03 \quad 9.6 \%$

55CS $137 \quad 3.935 E-03 \quad 2.3 \%$

57LA $140 \quad 7.716 \mathrm{E}+01 \quad 9.5 \%$

63EU $154 \quad 3.423 E+03 \quad 1.7 \%$

60. 8-MAY-1996 9:50:14 -48177 .

$11 \mathrm{NA} 24 \quad 3.820 \mathrm{E}-06 \quad 117.1 \%$

4OZR $95 \quad 6.669 E+03 \quad 16.3 \%$

44RU $105 \quad 3.976 \mathrm{E}+04 \quad 5.6 \%$

$55 \mathrm{CS} 137 \quad 1.993 E-02 \quad 3.4 \%$

60. 8-MAY-1996 $9: 51: 20 \quad-49323$,

$80 \quad 19 \quad 1.495 E+02 \quad 57.0 \%$

23V $52 \quad 8.315 E+01 \quad 39.0 \%$

4OZR $95 \quad 1.084 E+04 \quad 18.6 \%$

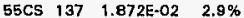

90THTH $232 \quad 6.695 \mathrm{E}-02 \quad 12.0 \%$

60. 8-MAY-1996 9:52:59 -50017 .

$11 \mathrm{NA} 22 \quad 4.854 \mathrm{E}+01 \quad \mathrm{~B} .1 \%$

$27 \mathrm{CO} \quad 60 \quad 3.190 \mathrm{E}+01 \quad 10.5 \%$

$46 P D \quad 109 \quad 2.387 E-03 \quad 32.8 \%$

$\begin{array}{lllll} & 109 & 2.360 E-03 & 32.8 \%\end{array}$

52TEM $127 \quad 4.432 \mathrm{E}-03 \quad 118.4 \%$

$55 \mathrm{CS} \quad 137 \quad 1.516 \mathrm{E}-03 \quad 2.0 \%$

57LA $140 \quad 4.997 E+00 \quad 31.4 \%$

63EU $154 \quad 1.374 \mathrm{E}+02 \quad 8.0 \%$

$92 \mathrm{U} 232$ 1.130E-02 118.4\%

60. 8.MAY-1996 9:54:08 -48849 .

$11 \mathrm{NA} 22 \quad 1.500 \mathrm{E}+03 \quad 2.3 \%$

$27 \mathrm{CO} \quad 60 \quad 5.246 \mathrm{E}+02 \quad 2.9 \%$

47AGM $108 \quad 3.750 E+03 \quad 9.5 \%$

52TEM $127 \quad 4.918 \mathrm{E}-02 \quad 99.5 \%$

$55 \mathrm{CS} \quad 137 \quad 3,382 \mathrm{E}-03 \quad 2.5 \%$

57LA $140 \quad$ 1.02OE + O2 7.8\%

63EU $154 \quad 4.224 E+03 \quad 2.3 \%$

$92 \mathrm{U} \quad 232 \quad 1.254 \mathrm{E}-01 \quad 99.5 \%$

60. 8-MAY-1996 9:55:17 -47685.

$11 \mathrm{NA} 22 \quad 1.891 \mathrm{E}+04 \quad 4.2 \%$

$25 \mathrm{MN} 56 \quad 1.488 E+02 \quad 152.9 \%$

$27 \mathrm{CO} \quad 60 \quad 6.024 \mathrm{E}+03 \quad 4.3 \%$

4OZR $95 \quad 1.528 E+04 \quad 33.3 \%$

52TEM $127 \quad 2.150 E+00 \quad 79.4 \%$

52TE $129 \quad 2.183 E-03 \quad 156.2 \%$

55CS $137 \quad 5.225 E-02 \quad 5.6 \%$

63EU $154 \quad 5,325 E+04 \quad 4.2 \%$

69TM $170 \quad 4.401 E-02 \quad 19.4 \%$

90TH 228 t.174E-01 $19.4 \%$

$92 \cup \quad 232 \quad 5.483 E+00 \quad 79.4 \%$

90 TH $232 \quad 5.772 \mathrm{E}+00 \quad 79.4 \%$

90 THTH232 1.174E-01 19.4\%

60. 8-MAY-1996 9:56:23 -48179.

$\begin{array}{lll}21 \mathrm{SC} & 48 & 4.489 \mathrm{E}+01 \quad 79.6 \%\end{array}$

40ZA $95 \quad 1.083 E+04 \quad 18.8 \%$

52TEM $127 \quad 4.206 \mathrm{E}-01 \quad 68.3 \%$

$\begin{array}{llll}56 B A & 133 & 6.6 B 5 E-04 & 45.1 \%\end{array}$

$55 \mathrm{CS} \quad 137 \quad 2.512 \mathrm{E}-02 \quad 5.4 \%$

69TM 170 3.168E-02 $13.4 \%$

90TH $228 \quad 8.452 \mathrm{E}-02 \quad 13,4 \%$

$92 \mathrm{U} \quad 232 \quad 1.072 \mathrm{E}+00 \quad 68.3 \%$ 
WHC-SD-W151-CN-001, Rev. 0

Page 24 of 29

A3650809570 50896 09:57:38

A3650809580 50896 09:58:46

A3650809590 $5089609: 59: 55$

A3650810010 50896 10:01:04

A3650810020 50896 10:02:10

A3650810030 50896 10:03:16

A.3650810040 50B96 10:04:23

A3650810050 50896 10:05:31

A3650810060 50896 10:06:39
90THTH232 8.452E-02 13.4\%

60. 8-MAY-1996 9:57:32 $\quad-49379$

11NA $22 \quad 6.151 E+03 \quad 1.5 \%$

$27 \mathrm{CO} \quad 60 \quad 2.008 E+03 \quad 3.0 \%$

47AGM $108 \quad 1.968 E+04 \quad 7.5 \%$

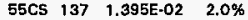

57LA $140 \quad 4.127 E+02 \quad 7.6 \%$

63EU $154 \quad 1.735 E+04 \quad 1.5 \%$

69TM $170 \quad 2,145 E-02 \quad 11.7 \%$

90TH $228 \quad 5.723 \mathrm{E}-02 \quad 11.7 \%$

90TKITH232 5.723E-02 $\quad 11.7 \%$

60. B-MAY-1996 9:58:38 $\quad-50535$.

IINA $22 \quad 4.974 E+09 \quad 8.0 \%$

$27 \mathrm{CO} \quad 60 \quad 2.261 E+01 \quad 10.0 \%$

$\begin{array}{llll}55 \mathrm{CS} & 137 & 1.364 \mathrm{E}-03 & 2.7 \%\end{array}$

G3EU $154 \quad 1.401 E+02 \quad 8.0 \%$

60. B-MAY-1996 $9: 59: 47 \quad-51730$.

IINA $22 \quad 1.662 E+03 \quad 2.6 \%$

$27 \mathrm{CO} \quad 60 \quad 5.929 \mathrm{E}+02 \quad 2.6 \%$

4OZR $95 \quad 1.077 E+03 \quad 32.3 \%$

47AGM $108 \quad 4.365 E+03 \quad 8.0 \%$

$55 \mathrm{CS} \quad 134 \quad 2.956 \mathrm{E}+01 \quad 41.7 \%$

55CS $137 \quad 4.436 \mathrm{E}-03 \quad 2.0 \%$

57LA $140 \quad 1.185 E+02 \quad 5.9 \%$

63EU $154 \quad 4.683 E+03 \quad 2.6 \%$

90 THTH $232 \quad 2.114 \mathrm{E}-02 \quad 11.8 \%$

60. 8-MAY-1996 10:00:56 .52939 .

$11 \mathrm{NA} 22 \quad 1.757 \mathrm{E}+03 \quad 2.5 \%$

$27 \mathrm{CO} \quad 60 \quad 6.286 \mathrm{E}+02 \quad 2,6 \%$

47AGM $108 \quad 5.093 E+03 \quad 7.8 \%$

$55 \mathrm{CS} \quad 137 \quad 5.048 \mathrm{E} \cdot 03 \quad 2.4 \%$

57LA $140 \quad 1.217 E+02 \quad 6.0 \%$

63EU $154 \quad 4.948 E+03 \quad 25 \%$

60. B-MAY-1996 10:02:05 -54000.

$11 \mathrm{NA} 22 \quad 1.990 \mathrm{E}+01 \quad 13.9 \%$ $27 C O \quad 60 \quad 1.448 E+01 \quad 15.3 \%$

$55 \mathrm{CS} \quad 137 \quad 7.369 \mathrm{E}-04 \quad 2.9 \%$

63EU $154 \quad 5.605 E+01 \quad 13.9 \%$

60. B-MAY-1996 10:03:11 -55294.

11NA $22 \quad 1.807 E+01 \quad 16.1 \%$ $20 \mathrm{CA} 47 \quad 3.053 E+00 \quad 71.3 \%$ $27 \mathrm{CO} \quad 60 \quad 1.442 E+01 \quad 18.2 \%$

$531 \quad 135 \quad 8.772 \mathrm{E}+00 \quad 59.9 \%$

55Cs $137 \quad 8.210 E-04 \quad 2.8 \%$ G3EU $154 \quad 4.685 E+01 \quad 15.2 \%$

60. 8.MAY-1996 10:04:17 $\quad-56437$.

IINA $22 \quad 1.682 E+0178.0 \%$ IBAR $41 \quad 2.244 E+00 \quad 57.2 \%$

$27 \mathrm{CO} \quad 60 \quad 1.386 E+01 \quad 13.9 \%$

49INM $116 \quad 2.645 E+00 \quad 57.2 \%$

55CS $137 \quad 8,463 E-04 \quad 3,1 \%$

G3EU $154 \quad 4.737 E+01 \quad 18.0 \%$

60. B-MAY-1996 10:05:23 -57598 .

$11 \mathrm{NA} 22 \quad 1.963 \mathrm{E}+01 \quad 15.1 \%$

$27 \mathrm{CO} \quad 60 \quad 1.050 E+01 \quad 14.7 \%$

$\begin{array}{llll}55 \mathrm{CS} & 137 & 7.575 \mathrm{E}-04 & 2.8 \%\end{array}$

G3EU $154 \quad 5.526 E+01 \quad 15.1 \%$

60. 8-MAY-1996 10:06:33 57854.

IINA $22 \quad 1.755 E+01 \quad 16.2 \%$

27 CO $60 \quad 1.319 E+01 \quad 13.4 \%$

$55 \mathrm{CS} \quad 137 \quad 6.532 \mathrm{E}-04 \quad 3.0 \%$

G3EU $154 \quad 4.716 \mathrm{E}+01 \quad 14.7 \%$

$65 T B \quad 160 \quad 5.588 E+00 \quad 55.8 \%$

60. 8-MAY-1996 10:07:39 -57854.

I1NA $22 \quad 2.293 \mathrm{E}+01 \quad 13.9 \%$

$27 \mathrm{CO} \quad 60 \quad 1.306 \mathrm{E}+01 \quad 15.8 \%$ 
WHC-SD-WI51-CN-001, Rev. 0

Page 25 of 29

\begin{tabular}{|c|c|c|c|c|c|c|}
\hline \multirow[t]{6}{*}{ A3650810070 } & $5089610: 07: 49$ & 60. & $\begin{array}{r}\text { 63EU } \\
\text { 8-MAY-1996 } 10\end{array}$ & $\begin{array}{c}154 \\
0: 08: 5\end{array}$ & $\begin{array}{r}6.457 E+01 \\
51 \quad-57854 .\end{array}$ & $13.9 \%$ \\
\hline & & & I1NA & 22 & $1.365 E+01$ & $17.1 \%$ \\
\hline & & & $27 \mathrm{CO}$ & 60 & $1.712 E+01$ & $10.5 \%$ \\
\hline & & & $55 \mathrm{cs}$ & 137 & $6.647 \mathrm{E}-04$ & $3.5 \%$ \\
\hline & & & 57LA & 140 & $2.060 E+\infty 0$ & $53.2 \%$ \\
\hline & & & 63EU & 154 & $3.845 E+01$ & $17.1 \%$ \\
\hline \multirow[t]{5}{*}{ A3650810100 } & $5089610: 10: 05$ & 60. & 8-MAY-1996 1C & $0: 11: 0$ & $06 \quad-56708$ & \\
\hline & & & $11 \mathrm{NA}$ & 22 & $1.815 E+01$ & $14.8 \%$ \\
\hline & & & $27 \mathrm{CO}$ & 60 & $9.776 E+00$ & $17.2 \%$ \\
\hline & & & $55 \mathrm{cs}$ & 137 & $7.624 E-04$ & $2.8 \%$ \\
\hline & & & 63EU & 154 & $5.112 E+01$ & $14.8 \%$ \\
\hline \multirow[t]{7}{*}{ A 3650810110} & $50896 \quad 10: 11: 14$ & 60. & 8-MAY- 1996 IC & $0: 12: 1$ & $15 \quad-55548$ & \\
\hline & & & $11 \mathrm{NA}$ & 22 & $1.767 E+01$ & $17.2 \%$ \\
\hline & & & $27 \mathrm{Co}$ & 60 & $1.547 E+01$ & $11.4 \%$ \\
\hline & & & 52TEN & M 127 & $1.519 \mathrm{E}-02$ & $39.5 \%$ \\
\hline & & & $55 \mathrm{Cs}$ & 137 & $8.572 E \cdot 04$ & $3.7 \%$ \\
\hline & & & G3EU & 154 & $4.974 E+01$ & $17.2 \%$ \\
\hline & & & 920 & 232 & $3.874 E-02$ & $39.5 \%$ \\
\hline \multirow[t]{6}{*}{ A3650810120 } & $50896 \quad 10: 12: 21$ & 60. & 8-MAY-1996 10 & $0: 13: 2$ & $21 \quad-55147$. & \\
\hline & & & $11 \mathrm{NA}$ & 22 & $1.625 E+01$ & $15.4 \%$ \\
\hline & & & $27 \mathrm{CO}$ & 60 & $1.865 E+01$ & $12.2 \%$ \\
\hline & & & $55 \mathrm{Cs}$ & 137 & B.423E-04 & $2,7 \%$ \\
\hline & & & 63EU & 154 & $4.574 E+01$ & $15.4 \%$ \\
\hline & & & $90 \pi \mathrm{TT}$ & TH232 & 2 $9.328 E-03$ & $15.6 \%$ \\
\hline \multirow[t]{5}{*}{ A3650810130 } & $5089610: 13: 29$ & 60. & 8-MAY-1996 10 & $0: 14: 3$ & $30 \quad .56345$ & \\
\hline & & & 11NA & 22 & $1.390 E+01$ & $17.3 \%$ \\
\hline & & & $27 \mathrm{CO}$ & 60 & $1,584 E+01$ & $11.3 \%$ \\
\hline & & & $55 \mathrm{cs}$ & 137 & $8.250 E-04$ & $3.0 \%$ \\
\hline & & & 63EU & 154 & $3,844 E+01$ & $15.6 \%$ \\
\hline \multirow[t]{4}{*}{ A3650810140 } & 50896 10:14:38 & 60. & 8-MAY-1996 1C & $0: 15: 3$ & -57559 & \\
\hline & & & $11 \mathrm{NA}$ & 22 & $1,407 E+01$ & $16.0 \%$ \\
\hline & & & $\begin{array}{l}27 \mathrm{co} \\
55 \mathrm{cs}\end{array}$ & $\begin{array}{r}60 \\
137\end{array}$ & $\begin{array}{l}1.438 E+01 \\
7.656 E-04\end{array}$ & $\begin{array}{l}12.8 \% \\
4.0 \%\end{array}$ \\
\hline & & & $63 E U$ & 154 & $3.616 E+01$ & $15.1 \%$ \\
\hline \multirow[t]{6}{*}{ A3650810150 } & 50896 10:15:45 & 60. & 8.MAY-1996 IC & $0: 16: 4$ & $45 \quad .58704$ & \\
\hline & & & $11 \mathrm{NA}$ & 22 & $1.854 E+01$ & $16.7 \%$ \\
\hline & & & $27 \mathrm{CO}$ & 60 & $1,155 E+01$ & $13.7 \%$ \\
\hline & & & 5311 & 1355 & $5.380 E+\infty 0$ & $60.0 \%$ \\
\hline & & & $55 \mathrm{cs}$ & 137 & $5.706 E-04$ & $3.5 \%$ \\
\hline & & & 63EU & 154 & $5.221 E+01$ & $16.7 \%$ \\
\hline \multirow[t]{10}{*}{ A3650810170 } & 50896 10:17:02 & 60. & 8-MAY-1996 10 & $0: 18: 0$ & $04 \quad .60085$ & \\
\hline & & & $11 N A$ & 22 & $3.155 E+02$ & $2.1 \%$ \\
\hline & & & $27 \mathrm{CO}$ & 60 & $2.739 \mathrm{E}+02$ & $3.4 \%$ \\
\hline & & & 38SR & 92 & $2.893 E+00$ & $46.9 \%$ \\
\hline & & & $47 A G 1$ & M 108 & $32.304 E+03$ & $39.1 \%$ \\
\hline & & & 52TEN & M 127 & $2.367 \mathrm{E}-02$ & $87.8 \%$ \\
\hline & & & $55 \mathrm{cs}$ & 137 & $1.985 E \cdot 03$ & $1.9 \%$ \\
\hline & & & 57LA & 140 & $4.740 \mathrm{E}+01$ & $8.5 \%$ \\
\hline & & & 63EU & 154 & $2.021 E+03$ & $2.0 \%$ \\
\hline & & & 920 & 232 & $6.036 \mathrm{E}-02$ & $87.8 \%$ \\
\hline
\end{tabular}


WHC-SD-W151-CN-001, Rev. 0

Page 26 of 29

\subsection{CALCULATION}

All calculations are performed by the spread sheet. The ${ }^{137}$ Cs activity for every countering interval is calculated by equation:

$$
A=M A \times G F \times C_{F}
$$

where

$A$ is the calculated ${ }^{137} \mathrm{Cs}$ activity in $\mathrm{mCi}$.

MA is the activity of ${ }^{137} \mathrm{Cs}$ measured by the FRRDS.

$G F$ is the geometry correction factor ${ }^{3}$.

CF is the detector calibration factor provided by Radioanalytical Chemistry ${ }^{2}$.

Table 5.1 lists the correction factors for each detector.

TABLE 5.1: DETECTOR CORRECTION FACTORS

\begin{tabular}{|c|c|c|}
\hline Detector & GF & CF \\
\hline A1 & 1.481 & 1 \\
\hline A2 & 1.934 & 46 \\
\hline A3 & 1.957 & 1097000 \\
\hline
\end{tabular}

The total ${ }^{137} \mathrm{Cs}$ activity is obtained by summing the ${ }^{137} \mathrm{Cs}$ activity for all the individual segments. Because the thermocouple tree is long enough to extend into the sludge in the bottom of the tank, three values of ${ }^{137} \mathrm{Cs}$ activity are provided: ${ }^{137} \mathrm{Cs}$ activity for the 1 iquid phase, ${ }^{137} \mathrm{Cs}$ activity for the sludge phase, and ${ }^{137} \mathrm{Cs}$ activity for the entire thermocouple tree length. These values are used to calculate the inventory of the other radioisotopes using the distribution provided by Solid Waste Engineering ${ }^{1}$.

Solid Waste Engineering requires the data be reported in two ways. First, the activity of each isotope is divided by the volume of the shipping container, $3.624 \mathrm{~m}^{3}$, and given in curies per cubic meter. Then the activity of each isotope is divided by the mass of the thermocouple tree, 133,930 grams, and given in nanocuries per gram. 


\subsection{RESULTS}

The results are reported in tables 6.1 and 6.2

TABLE 6.1: TOTAL RADIONUCLIDE INVENTORY BASED ON LIQUID AND SLUDGE DISTRIBUTIONS Calculation of Isotopes in the Liquid Phase sludge Phase

$\begin{array}{lll}\text { nuclide } & \begin{array}{c}\text { ratio } \\ \text { nuclide/Cs-137 }\end{array} & \text { Curies } \\ \text { H-3 } & 1.78 E-05 & 5.17 E-06 \\ \text { C-14 } & 1.01 E-06 & 2.94 E-07 \\ \text { Co-60 } & 7.11 E-04 & 2.07 E-04 \\ \text { Se-79 } & 1.68 E-06 & 4.88 E-07 \\ \text { Y-90 } & 8.01 E-04 & 2.33 E-04 \\ \text { Sr-90 } & 8.01 E-04 & 2.33 E-04 \\ \text { Tc-99 } & 2.49 E-04 & 7.24 E-05 \\ \text { Ru-106 } & 7.07 E-04 & 2.06 E-04 \\ \text { Sb-125 } & 6.83 E-03 & 1.99 E-03 \\ \text { I-129 } & 4.68 E-07 & 1.36 E-07 \\ \text { CS-134 } & 3.14 E-03 & 9.13 E-04 \\ \text { Cs-137 } & 1.00 E+00 & 2.91 E-01 \\ \text { Ba-137m } & 9.46 E-01 & 2.75 E-01 \\ \text { Ce-144 } & 6.42 E-05 & 1.87 E-05 \\ \text { EU-154 } & 2.67 E-03 & 7.76 E-04 \\ U-234 & 4.03 E-07 & 1.17 E-07 \\ U-235 & 1.34 E-08 & 3.90 E-09 \\ U-236 & 3.38 E-08 & 9.83 E-09 \\ U-238 & 2.37 E-07 & 6.89 E-08 \\ \text { Np-237 } & 4.60 E-08 & 1.34 E-08 \\ \text { PU-238 } & 4.03 E-08 & 1.17 E-08 \\ \text { PU-239 } & 4.04 E-08 & 1.17 E-08 \\ \text { PU-240 } & 4.04 E-08 & 1.17 E-08 \\ \text { PU-241 } & 2.65 E-08 & 7.70 E-09 \\ \text { Am-241 } & 3.15 E-05 & 9.16 E-06 \\ \text { Cm-242 } & 2.52 E-12 & 7.33 E-13 \\ \text { Cm-243 } & 2.47 E-08 & 7.18 E-09 \\ \text { Cm-244 } & 3.99 E-07 & 1.16 E-07\end{array}$

$\begin{array}{cl}\begin{array}{c}\text { ratio } \\ \text { nuclide/Cs-137 }\end{array} & \text { Curies } \\ 8.88 E-06 & \\ 1.35 E-06 & 3.21 E-08 \\ 3.01 E-03 & 4.89 E-09 \\ 2.76 E-06 & 1.09 E-05 \\ 3.50 E+01 & 9.99 E-09 \\ 3.50 E+01 & 1.27 E-01 \\ 3.28 E-03 & 1.27 E-01 \\ 4.28 E-02 & 1.19 E-05 \\ 1.44 E-01 & 5.21 E-04 \\ 4.75 E-05 & 1.72 E-04 \\ 2.49 E-03 & 9.01 E-06 \\ 1.00 E+00 & 3.62 E-03 \\ 9.46 E-01 & 3.42 E-03 \\ 2.21 E-02 & 8.00 E-05 \\ 1.13 E-01 & 4.09 E-04 \\ 3.81 E-06 & 1.38 E-08 \\ 1.27 E-07 & 4.60 E-10 \\ 3.22 E-07 & 1.17 E-09 \\ 2.26 E-06 & 8.18 E-09 \\ 6.97 E-05 & 2.52 E-07 \\ 4.72 E-04 & 1.71 E-06 \\ 3.12 E-03 & 1.13 E-05 \\ 8.78 E-04 & 3.18 E-06 \\ 3.22 E-02 & 1.17 E-04 \\ 3.16 E-01 & 1.14 E-03 \\ 6.56 E-09 & 2.37 E-11 \\ 4.31 E-05 & 1.56 E-07 \\ 7.00 E-04 & 2.53 E-06\end{array}$

curies $/ m^{\wedge} 3$

nci/gm

1. $44 \mathrm{E}-06$

8. $24 \mathrm{E}-08$

6. $00 E-05$

$1.38 E-07$

3.50E-02

$3.50 E-02$

2.33E-05

$9.95 \mathrm{E}-05$

6.92E-04

$8.50 E-08$

2.54E-04

8. $12 \mathrm{E}-02$

7.68E-02

2.72E-05

3.27E-04

$3.61 \mathrm{E}-08$

1.20E-09

3. $03 \mathrm{E}-09$

2. $13 \mathrm{E}-08$

7.33E-08

4. $75 \mathrm{E}-07$

$3.12 \mathrm{E}-06$

8. $80 \mathrm{E}-07$

$3.22 E-05$

$3.18 \mathrm{E}-04$

6.75E-12

$4.50 \mathrm{E}-08$

$7.31 E-07$

3.89E-02

2. $23 \mathrm{E}-03$

$1.62 \mathrm{E}+00$

3.72E-03

$9.48 \mathrm{E}+02$

$9.48 \mathrm{E}+02$

$6.29 \mathrm{E}-01$

$2.69 \mathrm{E}+00$

$1.87 E+01$

2.30E-03

$6.88 \mathrm{E}+00$

2. $20 \mathrm{E}+03$

2. $08 \mathrm{E}+03$

7.37E-01

$8.85 \mathrm{E}+00$

$9.78 \mathrm{E}-04$

$3.25 \mathrm{E}-05$

8.21E-05

5.76E-04

$1.98 \mathrm{E}-03$

1.28E-02

8.44E-02

2.38E-02

8.70E-01

$8.61 E+00$

$1.83 E-07$

$1.22 \mathrm{E}-03$

$1.98 \mathrm{E}-02$ 
WHC-SD-W151-CN-001, Rev, 0

Page 28 of 29

TABLE 6.2: TOTAL RADIONUCLIDE INVENTORY BASED ON AVERAGE DISTRIBUTION

\begin{tabular}{|c|c|c|c|c|}
\hline nuclide & $\begin{array}{c}\text { ratio } \\
\text { nuctide/Cs-137 }\end{array}$ & Curies & curies $/ m^{\wedge} 3$ & $\mathrm{nci} / \mathrm{gm}$ \\
\hline $\begin{array}{l}H-3 \\
C-14 \\
C o-60 \\
S e-79 \\
Y-90 \\
S r-90 \\
T c-99 \\
R u-106 \\
S b-125 \\
I-129 \\
C s-134 \\
C s-137 \\
B a-137 m \\
C e-144 \\
\text { Eu-154 } \\
U-234 \\
U-235 \\
U-236 \\
U-238 \\
N P-237 \\
P U-238 \\
P U-239 \\
P u-240 \\
P U-241 \\
\text { Am-241 } \\
\text { Cm-242 } \\
\text { Cm-243 } \\
\text { Cm-244 }\end{array}$ & $\begin{array}{l}1.73 E-05 \\
1.03 E-06 \\
8.39 E-04 \\
1.74 E-06 \\
1.96 E+00 \\
1.96 E+00 \\
4.19 E-04 \\
3.06 E-03 \\
1.45 E-02 \\
3.10 E-06 \\
3.10 E-03 \\
1.00 E+00 \\
9.46 E-01 \\
1.30 E-03 \\
8.84 E-03 \\
5.94 E-07 \\
1.98 E-08 \\
4.99 E-08 \\
3.50 E-07 \\
3.94 E-06 \\
2.64 E-05 \\
1.75 E-04 \\
4.92 E-05 \\
1.80 E-03 \\
1.77 E-02 \\
3.70 E-10 \\
2.43 E-06 \\
3.96 E-05\end{array}$ & $\begin{array}{l}5.09 \mathrm{E}-06 \\
3.03 \mathrm{E}-07 \\
2.47 \mathrm{E}-04 \\
5.12 \mathrm{E}-07 \\
5.77 \mathrm{E}-01 \\
5.77 \mathrm{E}-01 \\
1.23 \mathrm{E}-04 \\
9.01 \mathrm{E}-04 \\
4.27 \mathrm{E}-03 \\
9.12 \mathrm{E}-07 \\
9.12 \mathrm{E}-04 \\
2.94 \mathrm{E}-01 \\
2.78 \mathrm{E}-01 \\
3.83 \mathrm{E}-04 \\
2.60 \mathrm{E}-03 \\
1.75 \mathrm{E}-07 \\
5.83 \mathrm{E}-09 \\
1.47 \mathrm{E}-08 \\
1.03 \mathrm{E}-07 \\
1.16 \mathrm{E}-06 \\
7.77 \mathrm{E}-06 \\
5.15 \mathrm{E}-05 \\
1.45 \mathrm{E}-05 \\
5.30 \mathrm{E}-04 \\
5.21 \mathrm{E}-03 \\
1.09 \mathrm{E}-10 \\
7.15 \mathrm{E}-07 \\
1.17 \mathrm{E}-05\end{array}$ & $\begin{array}{l}1.41 \mathrm{E}-06 \\
8.37 \mathrm{E}-08 \\
6.81 \mathrm{E}-05 \\
1.41 \mathrm{E}-07 \\
1.59 \mathrm{E}-01 \\
1.59 \mathrm{E}-01 \\
3.40 \mathrm{E}-05 \\
2.49 \mathrm{E}-04 \\
1.18 \mathrm{E}-03 \\
2.52 \mathrm{E}-07 \\
2.52 \mathrm{E}-04 \\
8.12 \mathrm{E}-02 \\
7.68 \mathrm{E}-02 \\
1.06 \mathrm{E}-04 \\
7.18 \mathrm{E}-04 \\
4.82 \mathrm{E}-08 \\
1.61 \mathrm{E}-09 \\
4.05 \mathrm{E}-09 \\
2.84 \mathrm{E}-08 \\
3.20 \mathrm{E}-07 \\
2.14 \mathrm{E}-06 \\
1.42 \mathrm{E}-05 \\
4.00 \mathrm{E}-06 \\
1.46 \mathrm{E}-04 \\
1.44 \mathrm{E}-03 \\
3.01 \mathrm{E}-11 \\
1.97 \mathrm{E}-07 \\
3.22 \mathrm{E}-06\end{array}$ & $\begin{array}{l}3.80 \mathrm{E}-02 \\
2.26 \mathrm{E}-03 \\
1.84 \mathrm{E}+00 \\
3.82 \mathrm{E}-03 \\
4.31 \mathrm{E}+03 \\
4.31 \mathrm{E}+03 \\
9.21 \mathrm{E}-01 \\
6.72 \mathrm{E}+00 \\
3.19 \mathrm{E}+01 \\
6.81 \mathrm{E}-03 \\
6.81 \mathrm{E}+00 \\
2.20 \mathrm{E}+03 \\
2.08 \mathrm{E}+03 \\
2.86 \mathrm{E}+00 \\
1.94 \mathrm{E}+01 \\
1.31 \mathrm{E}-03 \\
4.35 \mathrm{E}-05 \\
1.10 \mathrm{E}-04 \\
7.69 \mathrm{E}-04 \\
8.66 \mathrm{E}-03 \\
5.80 \mathrm{E}-02 \\
3.85 \mathrm{E}-01 \\
1.08 \mathrm{E}-01 \\
3.96 \mathrm{E}+00 \\
3.89 \mathrm{E}+01 \\
8.13 \mathrm{E}-07 \\
5.34 \mathrm{E}-03 \\
8.70 \mathrm{E}-02\end{array}$ \\
\hline
\end{tabular}

\subsection{CONCLUSIONS}

7.1 Solid Waste Engineering is provided with the results to determine the waste classification.

7.2 The three detectors operated as expected providing good correlation of ${ }^{137} \mathrm{Cs}$ activity along the length of the thermocouple tree. 
WHC-SD-W151-CN-001, Rev. 0

Page 29 of 29

\subsection{REFERENCES}

1. Memo, D. C. Hetzer to P. A. Gagnon, "Radionuclide Distribution for the 101-AZ Waste Tank W-151 Disposal Project", 77520-95-073, dated August 29, 1995.

2. cc:Mail, G. L. Troyer to S. F. Kessler, "Thermocouple 13D Gamma Assay Data - Final", dated February 15, 1995, 9:18 a.m.

3. Memo, J. V. Nelson to E. M. Nordquist, "Recommendation on Configuring the Flexible Receiver Radiation Detection Assessing Residual Contamination on Thermocouple Instrument Trees Pulled from Tank 101-AZ", dated November 15, 1995. 


\begin{tabular}{|c|c|c|c|c|c|}
\hline \multicolumn{6}{|c|}{ DISTRIBUTION SHEET } \\
\hline To & \multirow{2}{*}{\multicolumn{3}{|c|}{$\begin{array}{l}\text { From } \\
\text { Criticality and Shielding }\end{array}$}} & \multicolumn{2}{|c|}{ Page 1 of 1} \\
\hline Distribution & & & & \multicolumn{2}{|c|}{ Date $05 / 23 / 96$} \\
\hline \multirow{2}{*}{\multicolumn{4}{|c|}{$\begin{array}{l}\text { Project Title/Work Order } \\
\text { Radioisotope Inventory of the T10IAZ Thermocouple Tree From } \\
\text { Riser 13D }\end{array}$}} & \multicolumn{2}{|c|}{ EDT No. 616553} \\
\hline & & & & \multicolumn{2}{|c|}{ ECN No. } \\
\hline Name & MSIN & $\begin{array}{l}\text { Text } \\
\text { With All } \\
\text { Attach. }\end{array}$ & Text Only & $\begin{array}{l}\text { Attach.f } \\
\text { Appendix } \\
\text { Only }\end{array}$ & $\begin{array}{l}\text { EDT/ECN } \\
\text { Only }\end{array}$ \\
\hline M. D. Ellefson & $56-30$ & $x$ & & & - \\
\hline P. A. Gagnon & $S 6-31$ & $x$ & & & \\
\hline J. G. Greenborg & $\mathrm{HO}-35$ & $x$ & & & \\
\hline D. C. Hetzer & S6-31 & $x$ & & & \\
\hline S. F. Kessier & HO-35 & $x$ & & & \\
\hline E. M. Nordquist & $S 2-48$ & $x$ & & & \\
\hline A. L. Ramble & $A 3-38$ & $x$ & & & \\
\hline H. L. Roach & $H 5-57$ & $x$ & & & \\
\hline G. L. Troyer & $T 6-50$ & $x$ & & & \\
\hline Project Files & $\mathrm{H} 6-08$ & $x$ & & & \\
\hline Central Files (Original +3 ) & $A 3-88$ & $x$ & & & \\
\hline
\end{tabular}

